\title{
Astrophysical science metrics for next-generation gravitational-wave detectors
}

\author{
R. X Adhikari ${ }^{1}$, P. Ajith ${ }^{2}$, Y. Chen ${ }^{3}$, J. A. Clark ${ }^{4}$, V. Dergachev ${ }^{5}$, \\ N. V. Fotopoulos ${ }^{1}$, S. E. Gossan ${ }^{1}$, I. Mandel ${ }^{6,7,8}$, M. Okounkova ${ }^{3}$, \\ V. Raymond $^{9}$, J. S. $\operatorname{Read}^{10}$ \\ E-mail: rana@caltech.edu \\ ${ }^{1}$ LIGO Laboratory, California Institute of Technology, Pasadena, CA 91125 USA \\ 2 International Centre for Theoretical Sciences, Tata Institute of Fundamental Research, \\ Bangalore 560089, India \\ 3 TAPIR, California Institute of Technology, Pasadena, CA 91125 USA \\ ${ }^{4}$ Georgia Tech, Atlanta, GA USA \\ 5 Max-Planck-Institut für Gravitationphysik, Callinstrasse 38, 30167, Hannover, Germany \\ ${ }^{6}$ Institute for Gravitational Wave Astronomy and School of Physics and Astronomy, \\ University of Birmingham, Edgbaston, Birmingham B15 2TT, United Kingdom \\ ${ }^{7}$ Monash Centre for Astrophysics, School of Physics and Astronomy, Monash University, \\ Clayton, Victoria 3800, Australia \\ ${ }^{8}$ OzGrav, Australian Research Council Centre of Excellence for Gravitational Wave \\ Discovery \\ ${ }^{9}$ Cardiff School of Physics and Astronomy, Cardiff University, Queens Buildings, The \\ Parade, Cardiff CF24 3AA, UK \\ ${ }^{10}$ California State University, Fullerton, CA USA
}

\begin{abstract}
The second generation of gravitational-wave detectors are being built and tuned all over the world. The detection of signals from binary black holes is beginning to fulfil the promise of gravitational-wave astronomy. In this work, we examine several possible configurations for third-generation laser interferometers in existing km-scale facilities. We propose a set of astrophysically motivated metrics to evaluate detector performance. We measure the impact of detector design choices against these metrics, providing a quantitative cost-benefit analyses of the resulting scientific payoffs.
\end{abstract}

\section{Contents}

1 Introduction 2

2 Interferometer Design 2

2.1 Sensitivity Limits of Second Generation Detectors . . . . . . . . . . . . . . 3

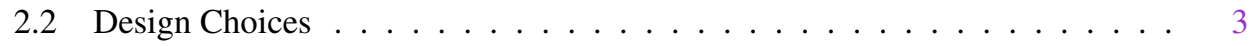

3 Astrophysical Metrics 5

3.1 Astrophysics with Compact Binary Coalescence . . . . . . . . . . . . . . 6

3.1.1 Binary Parameters and Populations. . . . . . . . . . . . . . 7

3.1.2 Intermediate Mass Black Holes . . . . . . . . . . . . . . . 11

3.1.3 Testing General Relativity. . . . . . . . . . . . . . . . . . 12

3.1.4 Dense Matter Equation of State from the Tidal Deformation of Neutron Stars . . . . . . . . . . . . . . . 15 
3.1.5 Measurement of the Cosmological Expansion . . . . . . . . . . . . 17

3.2 Astrophysics with Stellar Collapse . . . . . . . . . . . . . . . . 18

3.2.1 Detection Prospects. . . . . . . . . . . . . . . 20

3.2.2 Probing Core-Collapse Supernova Physics. . . . . . . . . . . . . . 20

3.2.3 Gravitational-Wave Memory in Stellar Collapse in the Milky Way . . 21

3.3 Astrophysics with Neutron Stars . . . . . . . . . . . . . . . . . . . 21

3.3.1 Bursting Magnetars, Glitching Pulsars. . . . . . . . . . . . . . . . . 21

3.3.2 Continuous Sources of GWs: Spinning Deformed Neutron Stars. . . . 22

3.3.3 Postmerger Oscillation Signal . . . . . . . . . . . . . . . . 24

3.4 Stochastic Background and Unanticipated Discoveries . . . . . . . . . . 27

$\begin{array}{lll}\text { Appendix A Estimating Signal-To-Noise Ratios } & 29\end{array}$

$\begin{array}{lll}\text { Appendix B Interferometer Parameters } & 30\end{array}$

\section{Introduction}

The recent detections of gravitational-wave $(\mathrm{GW})$ signals from merging binary black holes and neutron stars are beginning to fulfil the promise of GW astronomy. The Advanced LIGO detectors observed GW from a coalescences of two $\sim 30 M_{\odot}$ binary black holes on 14 September, 2015 [1]. Further observations of binary black mergers [2] and a binary neutron star merger [3] followed during the first two observing runs. The two Advanced LIGO detectors in the U.S. and the Virgo detector in Italy are gradually approaching their design sensitivity, the KAGRA detector in Japan is coming on-line, and the third LIGO interferometer in India is expected to join the world-wide network around 2025.

Within the LIGO Scientific Collaboration (LSC), the Interferometer Working Groups have identified a set of design concepts for the next generation of interferometer (known as LIGO Voyager) in publicly available technical documents [4, 5] and a manuscript in preparation [6]. It is expected that the following decades would see the development of new facilities supporting the proposed Einstein Telescope [7] and/or Cosmic Explorer [8] observatories.

In this work, we use the recently generated sensitivity curves to make quantitative estimates of the scientific potential of LIGO Voyager. In particular, we construct a Jacobian (cf. Tables $2 \& 3$ ), which relates the scientific outputs with changes to the interferometer's parameters. This Jacobian will help to make design trade-offs as the LIGO Voyager design moves forward. In Section 2, the design parameters of the interferometer are explained and the sensitivity curves shown. In Section 3, the astrophysical sources behind the Jacobian are introduced and the scientific metrics which form the rows of the Jacobian are described. The rest of the article describes the scientific targets from the various astrophysical sources.

\section{Interferometer Design}

The output of a workshop in January 2012 was a set of Strawman designs for a third generation LIGO [4] which have been iterated as new understanding arises. The three (Red, Green, and Blue) design teams worked to come up with separate designs for an interferometer that could be installed in the existing LIGO facilities without major facility modifications (i.e. keeping the same arm lengths, no modification of the $4 \mathrm{~km}$ beam tubes, etc.). Extra vacuum chambers, tubes, cryogenic equipment, and other vacuum equipment modifications were allowed for the purpose of this exercise. 


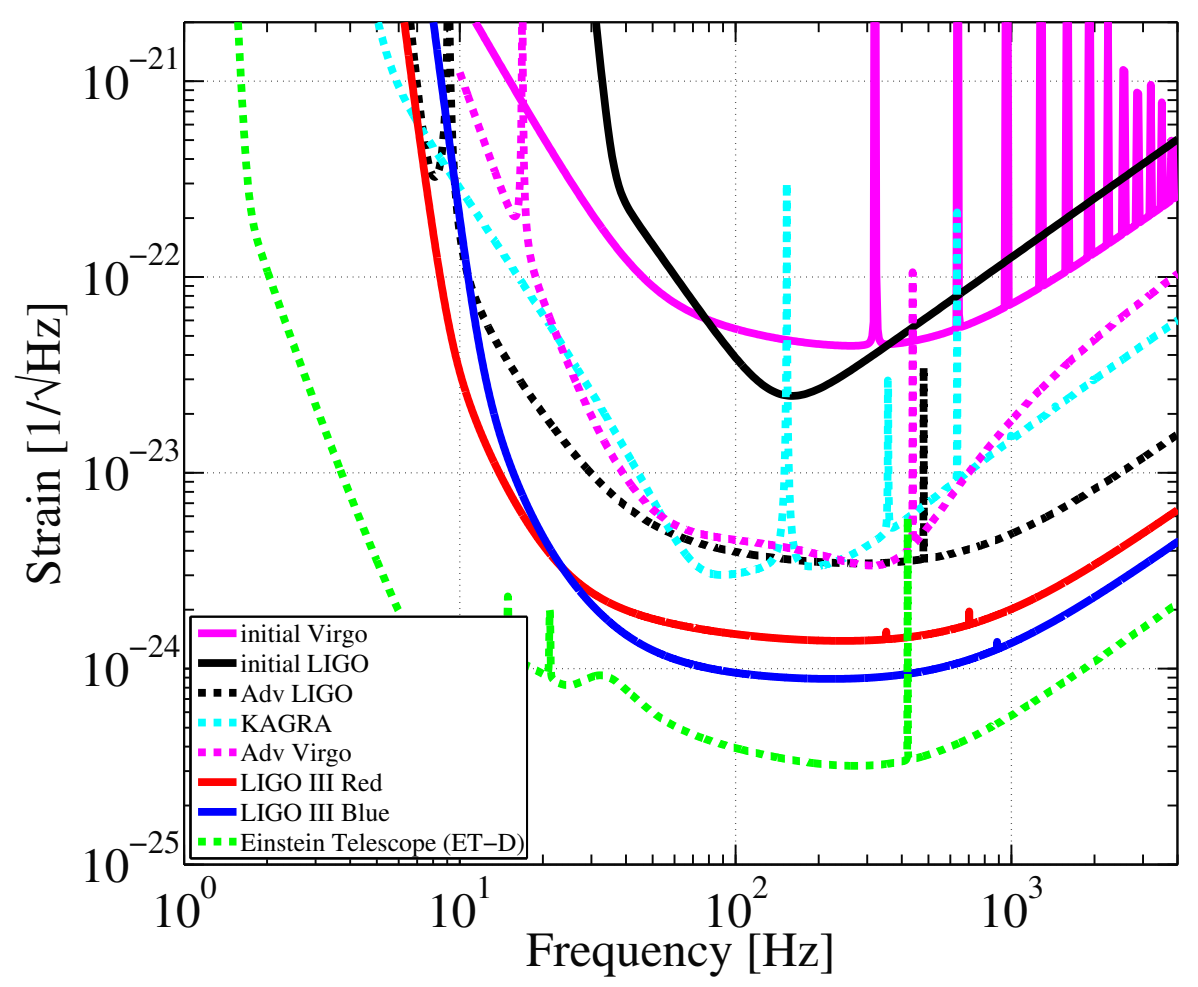

Figure 1: Shown are the strain noise spectral density estimates for first, second, and third generation detectors. The Red and Blue LIGO Voyager designs are shown here as well as the ET-D sensitivity estimate.

Within most of this document we only consider the Red and Blue designs. In particular, for the Jacobian table, we use the Blue design as the point of departure. However, as can be seen from Figure 1, the two designs are similar enough that this does not change the result too much. Throughout Section 3.1.1, all three design concepts are used to estimate scientific potential.

\subsection{Sensitivity Limits of Second Generation Detectors}

The second generation interferometers (Advanced LIGO, Advanced Virgo and KAGRA) all have similar noise limits. Figure 1 shows the estimated strain spectral density curves. The differences between the LIGO, Virgo, and KAGRA curves below $40 \mathrm{~Hz}$ arise from some uncertainty in the estimation of the true suspension thermal noise $[9,10]$ as well inherent differences in the baseline interferometer configurations chosen for the Virgo and KAGRA curves: the detuning leads to better sensitivity around $100 \mathrm{~Hz}$, but worse quantum noise performance at lower and higher frequencies. Note that these are just calculated noise curves and the true noise performance [11] of all detectors is likely to exceed these optimistic estimates in a few frequency bands.

\subsection{Design Choices}

In order to facilitate quantitative design choices, we have constructed the astrophysics and cosmology detector Jacobian tables and discuss the impact of various design changes on 


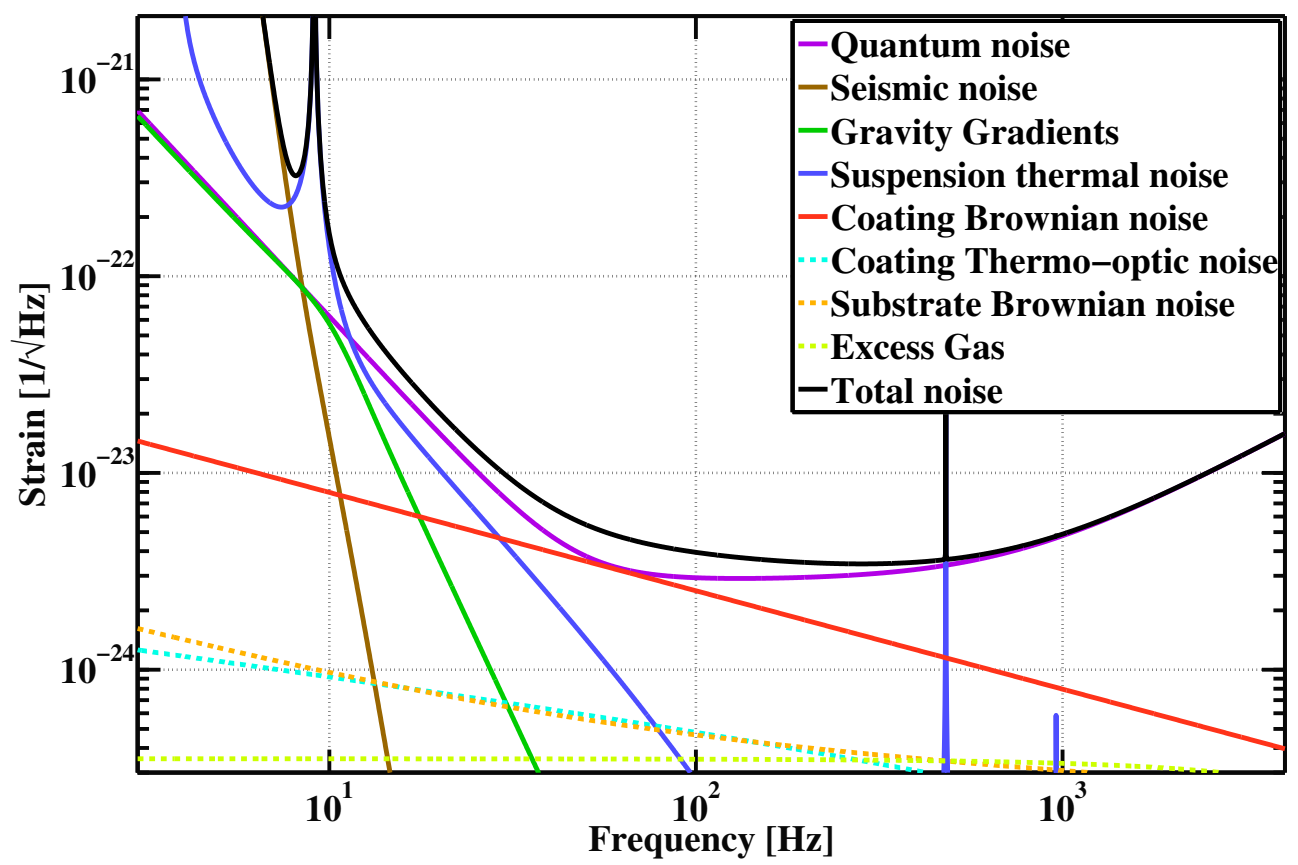

Figure 2: The Advanced LIGO[11] noise budget, computed using GWINC (120418)

astrophysical and cosmological science goals in Section 3. The columns in the Jacobian tables correspond to different interferometer design choices, while rows corresponds to different astrophysical figures of merit.

In all cases, the major cost is not monetary, rather it is the time spent in the installation and commissioning of these upgrades which must be considered when making the cost-benefit analysis of making the changes.

Here, we briefly describe the various columns in the detector Jacobians:

- NN: Fluctuations in the local Newtonian gravity field produce accelerations of the test mass which mask low frequency GW signals [13]. Reduction of this noise will require the expansion and improvement of the seismometer array used to estimate and subtract this noise offline.

- SEI: The motion of mirrors due to seismic disturbances in the $5-50 \mathrm{~Hz}$ band may be due to vibrations of the ground or the internal noise of the active seismic isolation systems [14]. Factors of a few reduction are possible using some few years of work on improving seismic sensors or redesigning the suspension configuration.

- SUS: The Brownian thermal noise of the mirror suspenion fibers is as significant at low frequencies as the seismic and gravity noise. Further development in suspension design [15] and materials science may lead to incremental progress in this band.

- SPOT/CTN: The limit to the interferometer sensitivity near $100 \mathrm{~Hz}$ is the Brownian thermal noise in the mirror coatings [16] for several different interferometer concepts. The power spectrum of this noise scales inversely with the mechanical quality factor $Q$ of the coating; it also scales inversely with the laser beam diameter. 


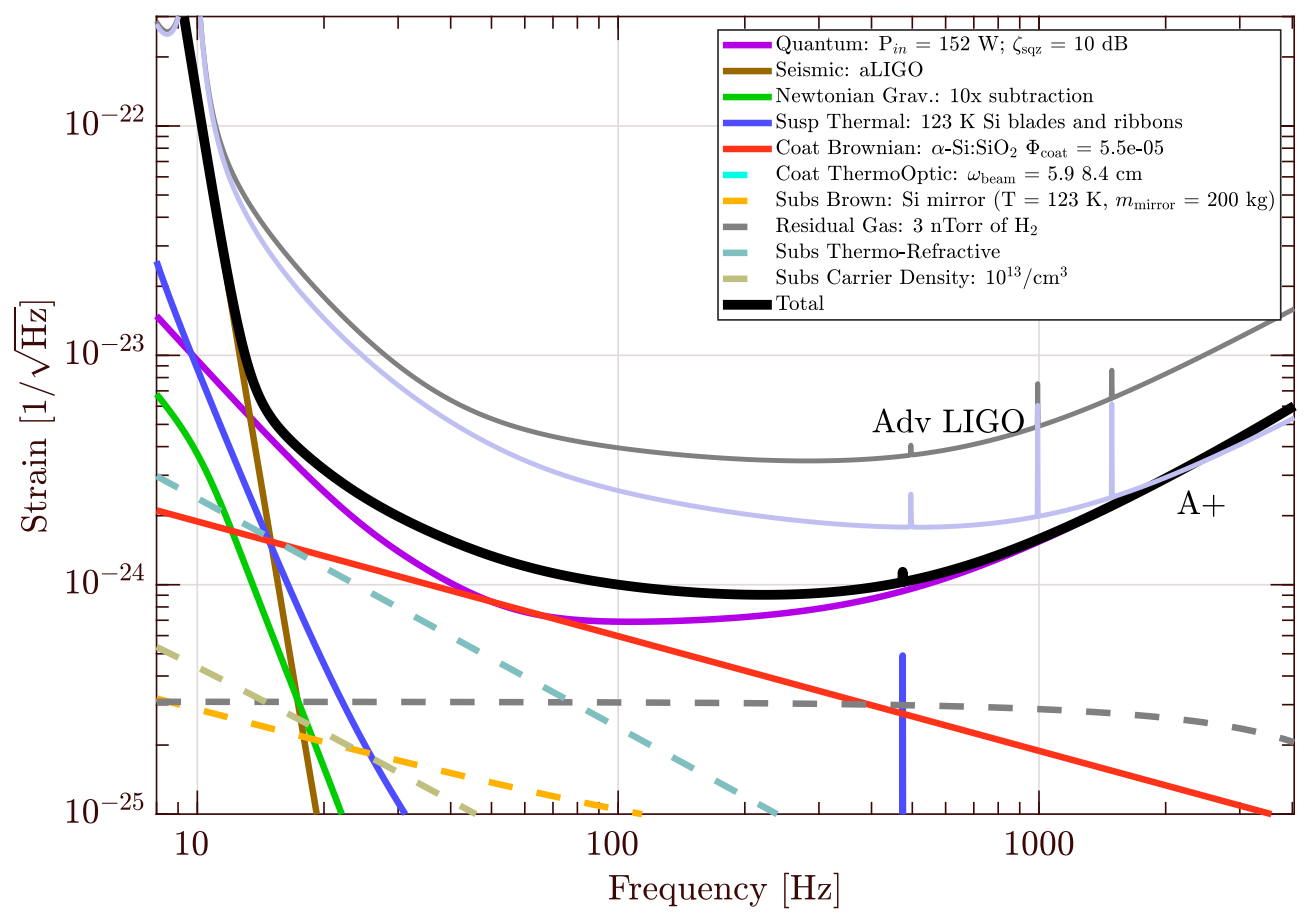

Figure 3: LIGO (Blue) Voyager Strain noise budget. Also shown are the Adv. LIGO design[11] and LIGO upgrade (a.k.a. A+)[12] noise curves. Comparison of interferometer physical parameters given in Appendix B.

- SQZ: Increasing the amount of squeezing delivered to the interferometer results in a broadband reduction of the quantum noise (both radiation pressure and shot noise). For technical reasons [17], this may have some frequency dependence but for the purposes of this analysis, we assume the naive broadband improvement.

- POW: Increasing the laser power mainly increases the low frequency radiation pressure noise and reduces the high frequency shot noise. When changing this parameter for the Jacobian table, we do not consider the thermal wavefront distortion effects due to the increased heat load on the mirrors.

- FCL: Increasing the length of the optical filter cavity [18], which is used to rotate the squeezing quadrature, reduces the degradation of the squeezed light which is injected into the interferometer dark port, and thereby improves the broadband sensitivity.

- MASS: A relatively simple way to reduce the quantum backaction noise (i.e. quantum radiation pressure) is to increase the mirror mass. In this column of the Jacobian, we do not consider the effects of increased mass on the suspension thermal noise.

\section{Astrophysical Metrics}

The main aim of this paper is to introduce a number of metrics to quantify the ability of the LIGO Voyager detectors to perform various astrophysical measurements, and study the variation of these figures of merit with respect to changes in different design parameters of the detector. Here we provide a brief overview of the astrophysical science that can be potentially 
performed by these detectors and to discuss figures of metrics related to these astrophysical measurements.

\subsection{Astrophysics with Compact Binary Coalescence}

Binaries of compact objects (black holes or neutron stars) can be produced in a number of astrophysical scenarios (cf. [19-22] for overviews). Once formed, they will radiate GWs, gradually shrinking the orbit through an inspiral that ends with the objects merging, then settling down into a spinning compact object. GWs emitted during the coalescence of binaries consisting of neutrons stars (NSs) and/or black holes (BHs, in the mass range from a few to $10^{3} M_{\odot}$ ) can be detected by ground-based GW observatories.

NS-NS and NS-BH binaries are typically expected to form through the evolution of isolated field binaries. An isolated binary composed of two main-sequence stars undergoes an evolution that involves several mass-transfer phases, possibly a common-envelope phase, and two core-collapse events of the binary's components. A review of the process, including the possible orderings of this sequence, is given in [23-25]. Occasionally, this process leaves behind a binary that is sufficiently compact to merge in a Hubble time through radiation reaction from $\mathrm{GW}$ emission (cf. [26-30]).

BH-BH binaries such as those responsible for the GW150914 event can evolve through isolated binary evolution as described above [e.g., 31-34], but could also be formed via chemically homogeneous evolution [35, 36], from primordial BHs [37, 38], or dynamically in dense stellar environments, such as globular clusters or nuclear clusters in galaxies [e.g., 3941]. There, a combination of three-body and four-body interactions, direct two-body capture, Kozai resonance and other dynamical effects can lead to the formation of coalescing binary BHs. These dynamical capture mechanisms could also drive mergers involving intermediatemass BHs (IMBHs), which are discussed in more detail in Section 3.1.2. The predicted merger rate for compact object binaries was uncertain, with plausible ranges spanning three orders of magnitude before the first GW detections [42]. LIGO observations have made it possible to constrain the binary black hole merger rate to a range spanning a factor of $\sim 10$ [2].

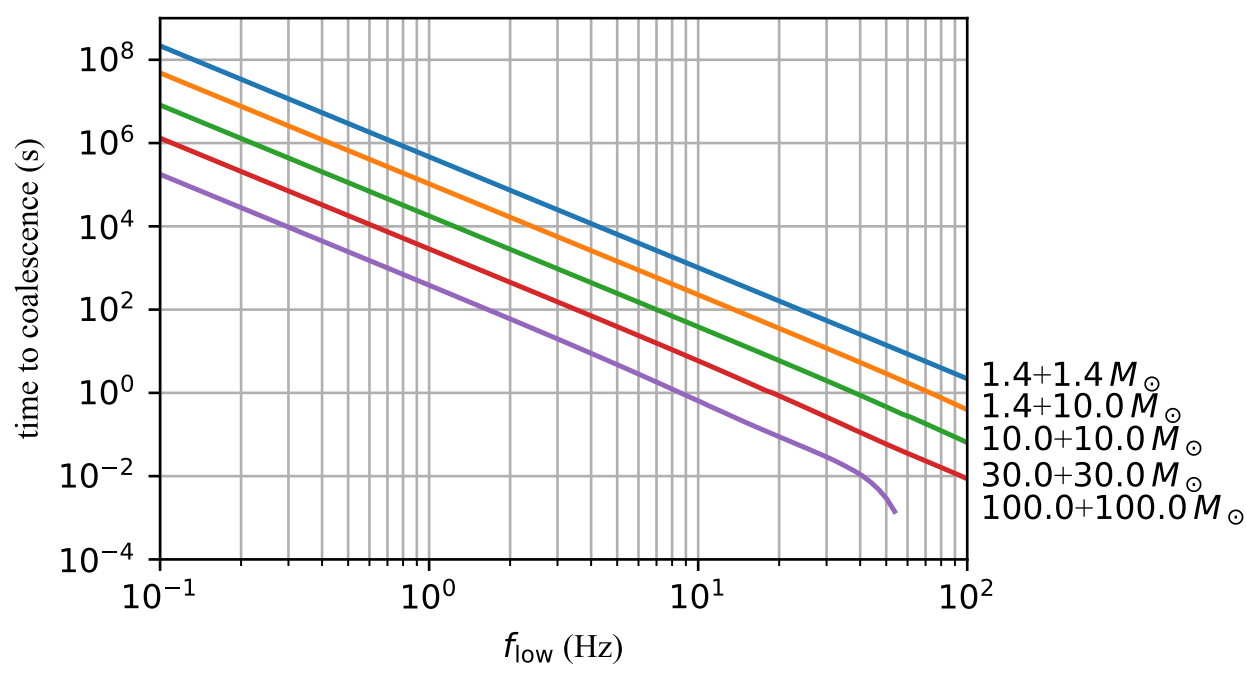

Figure 4: Time until merger, "chirp length", vs start frequency for NS/NS, NS/BH, and BH/BH systems. 


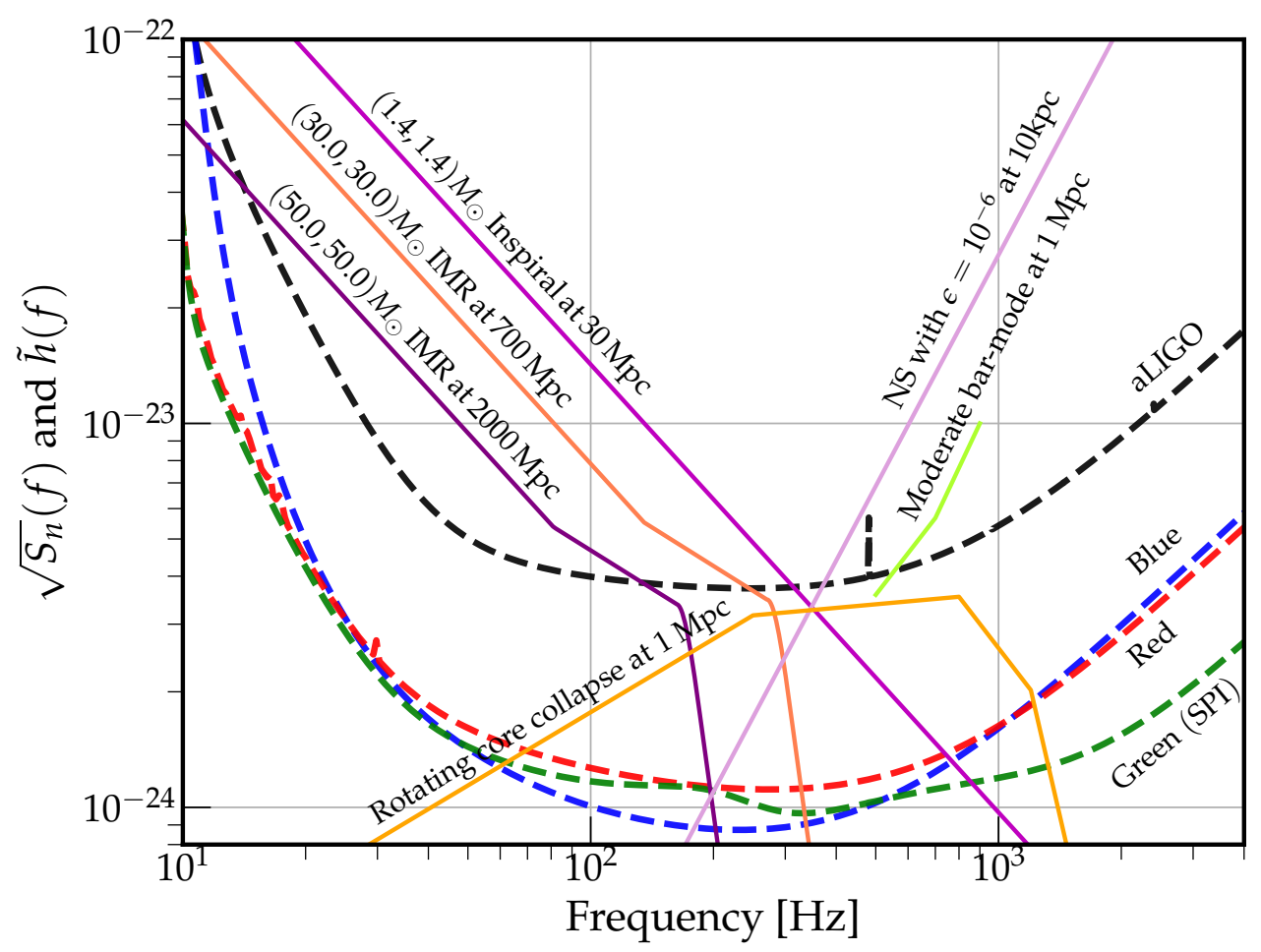

Figure 5: Baseline detector noise spectra compared with selected astrophysical sources.

The inspiral enters the detector's band when the orbital decay progresses to the point that the GW frequency (twice the orbital frequency for a circular system) is above the low frequency seismic "wall". As shown in Fig. 4, NS-NS waveforms will remain in band for many minutes with a low-frequency cut-off at $10 \mathrm{~Hz}$, though most of the signal-to-noise ratio (SNR) and bandwidth, which enable detection and parameter estimation, lie in the final seconds before merger (see Section 3.1.1 for a more detailed discussion). Binaries with a neutron star contain matter that can be ejected and fuel an electromagnetic counterpart, though potentially $\mathrm{BH}-$ BH binaries might also excite ambient material to become luminous. The discovery of an electromagnetic counterpart to the double neutron star merger GW170817 [43] led to an unprecedented observing campaign spanning all wavelengths, answering a number of longstanding questions, and posing a host of new ones.

3.1.1. Binary Parameters and Populations. GW observations of compact binary systems provide a great deal of information about the component objects as well as the populations of NSs and BHs in the Universe. The analysis of individual detections yields the masses and spins of the compact objects involved [44-46]. The distribution of these parameters in the population, along with the overall merger rates, will give critical insights into the processes that govern binary evolution. These include mass transfer in progenitors of compact binaries, supernova kicks, the efficiency of common-envelope ejection, and the dominant processes governing dynamical binary formation in dense stellar environments [e.g., 19, 24, 47, and references therein].

*The range of values correspond to different equations of states. 
CONTENTS

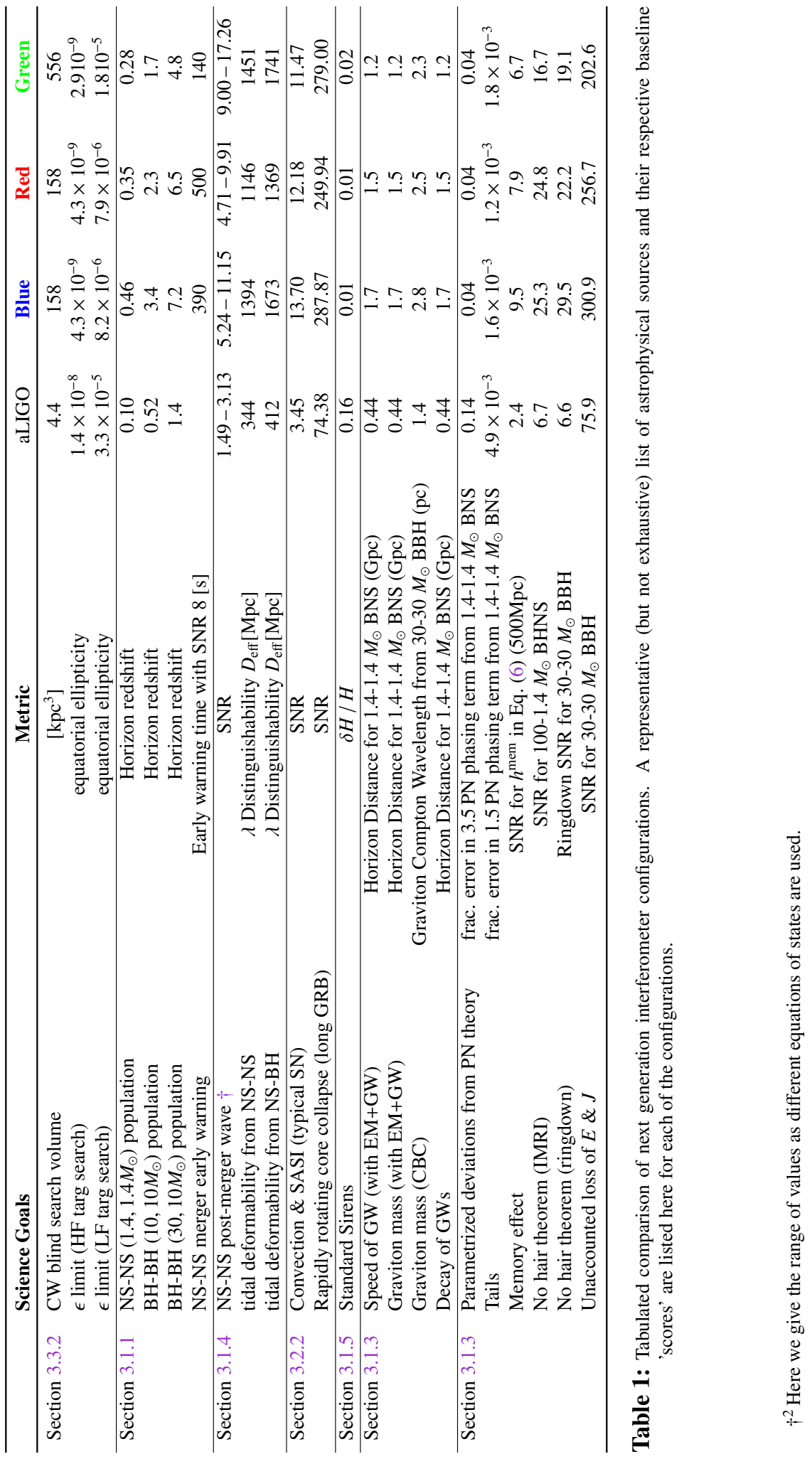




\begin{tabular}{llcccc}
\hline & Science Goals & NN & SEI & SUS & SPOT/CTN \\
\hline Section 3.3.2 & CW blind search volume & 0 & 0 & 0 & 0.04 \\
& $\epsilon$ limit (HF targ search) & 0 & 0 & 0 & -0.01 \\
& $\epsilon$ limit (LF targ search) & -0.05 & 0 & -0.13 & -0.14 \\
\hline Section 3.1.1 & NS-NS horizon & 0.01 & 0 & 0.02 & 0.21 \\
& BH-BH (10+10) horizon & 0.01 & 0 & 0.03 & 0.34 \\
& BH-BH (30+30) horizon & 0.02 & 0 & 0.04 & 0.09 \\
& CBC early warning & 0.11 & 0.09 & 0.22 & 0 \\
\hline Section 3.1.4 & NS-NS post-merger SNR * & 0 & 0 & 0 & $0-0.01$ \\
& tidal deformability from NS-NS & 0 & 0 & 0 & 0.06 \\
& tidal deformability from NS-BH & 0 & 0 & 0 & 0.01 \\
& NS f-mode 1590 Hz (SGR) & 0 & 0 & 0 & 0 \\
& NS mode 100-200 Hz (SGR) & 0 & 0 & 0 & 0.30 \\
\hline Section 3.2.2 & Convection \& SASI (typical SN) & 0 & 0 & 0 & 0.16 \\
& Rapidly rotating core collapse (long GRB) & 0 & 0 & 0 & 0.07 \\
\hline Section 3.1.5 & Standard Sirens & 0.01 & 0.01 & 0.03 & 0.53 \\
\hline Section 3.1.3 & Speed of GW (EM+GW) & 0.01 & 0.00 & 0.01 & 0.35 \\
& Graviton mass (EM+GW) & 0.01 & 0.00 & 0.01 & 0.35 \\
& Graviton mass (CBC) & 0.00 & 0.00 & 0.01 & 0.11 \\
& Decay of GWs & 0.01 & 0.00 & 0.01 & 0.35 \\
\hline Parametrized deviations from PN theory & 0.02 & 0.03 & 0.03 & 0.13 \\
& Tails & 0.04 & 0.06 & 0.06 & 0.15 \\
& Memory effect (BBH 30+30) & 0.01 & 0 & 0.01 & 0.34 \\
& No hair theorem (IMRI) & 0.02 & 0 & 0.05 & 0.27 \\
& No hair theorem (ringdown) & 0 & 0 & 0 & 0.18 \\
& Unaccounted loss of $E$ \& $J$ & 0.01 & 0.00 & 0.02 & 0.35 \\
\hline & & & & & \\
\hline
\end{tabular}

Table 2: Jacobian of Science goals as a function of interferometer upgrade technology: Each column corresponds to a configurable parameter or noise source: Newtonian noise (NN), seismic noise (SEI), suspension thermal noise at $10 \mathrm{~Hz}$ (SUS), coating thermal noise (CTN), and arm cavity laser beam spot size (SPOT). (Note that the sensitivity changes exactly the same way with respect to CTN and SPOT. Hence these quantities are shown in the same column). Each row corresponds to a particular science goal $\mathcal{S}$. Each element of the matrix is the logarithmic partial derivative $\partial \log \mathcal{S} / \partial x$ of the metric for a science goal $\mathcal{S}$ with respect to a parameter $x$ that represents a change in a particular interferometer component (e.g. laser power) or a component noise source (e.g. seismic noise). Both $\mathcal{S}$ and $x$ are normalized such that $\mathcal{S}=x=1$ for the 'baseline' detector configuration. Positive Jacobian elements correspond to detector changes which increase the SNR / decrease measurement errors.

Extracting this information will require a solution to the inverse problem of GW astrophysics: reconstructing the astrophysics from a collection of GW detections of coalescing binaries. This can be accomplished by a combination of two techniques: comparing observed distributions against modeled distributions predicted by population-synthesis simulations under a variety of assumptions [e.g., 48-50]; or model-independent efforts to distinguish subpopulations, such as those of dynamically formed black-hole binaries and evolved isolated binaries [e.g., 51-54].

For either approach, both source statistics and accurate estimates of the parameters of individual systems will be required. Here, we use the maximum (horizon) redshift at which a single detector could observe an optimally oriented, overhead source as a proxy for the amount of astrophysical knowledge that can be gained. Greater horizon redshifts increase the overall number of detections and make it possible to probe the evolution of merger rates and mass distributions with redshift; they also imply greater local sensitivity, increasing the number of high-SNR detections, where inference on component masses and spins will be most accurate. 


\begin{tabular}{llcccc}
\hline & Science Goals & SQZ & POW & FCL & MASS \\
\hline Section 3.3.2 & CW blind search volume & 1 & 0.3 & 0 & 0 \\
\hline & $\epsilon$ limit (HF targ search) & -0.24 & -0.08 & 0 & 0 \\
& $\epsilon$ limit (LF targ search) & -0.14 & -0.05 & 0.01 & -0.06 \\
\hline Section 3.1.1 & NS-NS horizon & 0.48 & 0.27 & -0.05 & 0 \\
& BH-BH (10+10) horizon & 0.75 & 0.42 & -0.07 & 0.01 \\
& BH-BH (30+30) horizon & 0.29 & 0.17 & -0.07 & 0.01 \\
& CBC early warning & 0.13 & -0.07 & 0.15 & 0.12 \\
\hline Section 3.1.4 & NS-NS post-merger SNR & 0.81 & 0.45 & 0 & 0 \\
& tidal deformability from NS-NS & 0.7 & 0.39 & 0 & 0 \\
& tidal deformability from NS-BH & 0.62 & 0.34 & 0.01 & 0 \\
& NS f-mode 1590 Hz (SGR) & 1.1 & 0.37 & 0.37 & 0.37 \\
& NS mode 100 - 200 Hz (SGR) & 0.08 & 0.07 & 0.12 & 0.11 \\
\hline Section 3.2.2 & Convection \& SASI (typical SN) & 0.58 & 0.32 & 0 & -0.01 \\
& Rapidly Rotating Core Collapse (long GRB) & 0.70 & 0.38 & 0 & 0 \\
\hline Section 3.1.5 & Standard Sirens & 0.52 & 0.35 & -0.05 & -0.38 \\
\hline Section 3.1.3 & Speed of GW (EM+GW) & 0.34 & 0.23 & -0.03 & -0.21 \\
& Graviton mass (EM+GW) & 0.34 & 0.23 & -0.03 & -0.21 \\
& Graviton mass (CBC) & 0.22 & 0.13 & 0.00 & -0.03 \\
& Decay of GWs & 0.34 & 0.23 & -0.03 & -0.21 \\
\hline Section 3.1.3 & Parametrized deviations from PN theory & 0.48 & 0.29 & -0.01 & -0.04 \\
& Tails & 0.38 & 0.23 & -0.02 & -0.04 \\
& Memory effect (BBH 30+30) & 0.38 & 0.24 & -0.02 & -0.19 \\
& No hair theorem (IMRI) & 0.32 & 0.21 & -0.05 & -0.10 \\
& No hair theorem (ringdown) & 0.66 & 0.36 & 0 & -0.01 \\
& Unaccounted loss of $E$ \& $J$ & 0.35 & 0.23 & -0.03 & -0.19 \\
\hline & & & & &
\end{tabular}

Table 3: Jacobian of Science goals as a function of interferometer upgrade technology (Table 2 continued): Each column corresponds to a configurable parameter or noise source: squeezing factor (SQZ), arm cavity stored power (POW), loss of the filter cavity for squeezing angle rotation (FCL), and mirror mass (MASS). Each row corresponds to a particular science goal $\mathcal{S}$. Each element of the matrix is the logarithmic partial derivative $\partial \log \mathcal{S} / \partial x$ of the metric for a science goal $\mathcal{S}$ with respect to a parameter $x$ that represents a change in a particular interferometer component (e.g. laser power) or a component noise source (e.g. seismic noise). Both $\mathcal{S}$ and $x$ are normalized such that $\mathcal{S}=x=1$ for the 'baseline' detector configuration. Positive Jacobian elements correspond to detector changes which increase the SNR / decrease measurement errors.

LIGO Voyager in any incarnation will have sensitivity to binary mergers at cosmological distances. For NS-NS binaries, the horizon redshift approaches $z \approx 0.5$, rising to $z \approx 7$ for BH-BH binaries with $30 M_{\odot}$ components. Hundreds or thousands of detections will allow us to precisely characterize the long-sought NS equation of state [55] and NS and $\mathrm{BH}$ mass and spin distributions in merging binaries. The equation of state measurement requires highfrequency sensitivity individually, but benefits also from a greater number of detections (cf. Section 3.3).

As mentioned earlier, multi-messenger observations are particularly exciting. These associate GWs from binary coalescences with coincident observations of the electromagnetic merger signatures, including detections with radio, optical, $\mathrm{x}$-ray and gamma-ray telescopes, and lowand high-energy neutrino detectors. For example, observations accompanying GW170817 have firmly established the relationship between gamma ray bursts and compact binary coalescences involving NSs [56].

The intriguing prospect of early-warning detection [57] is primarily dependent on low- 

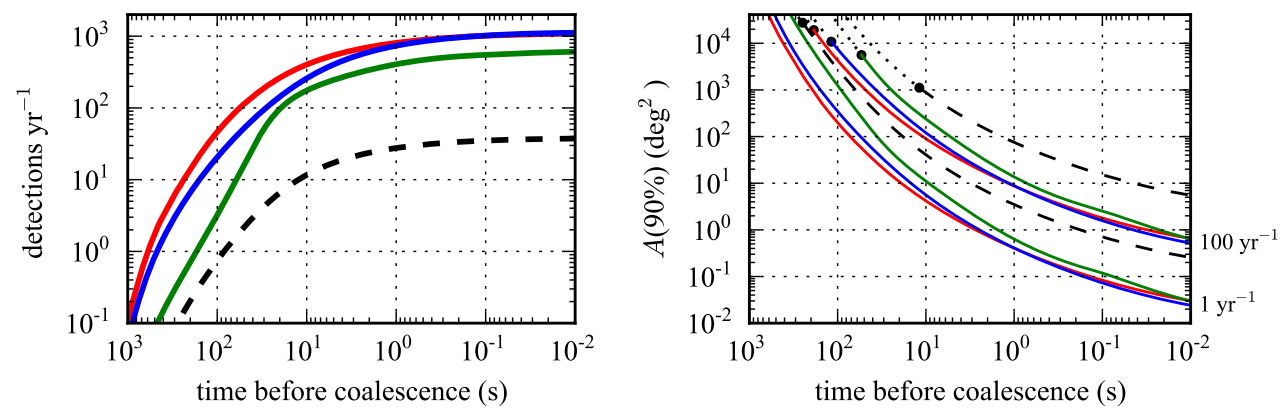

Figure 6: NS-NS early warning performance. Left: NS-NS detection rate per year $(\mathrm{SNR}=8)$ at times before merger, for an arbitrary reference merger rate density. Right: The evolving sky localization estimate at times before merger. For each detector, there are curves for two systems whose final amplitudes are set by their detection rates, labeled on the right. This plot assumes HLV as sites, but identical (LIGO) detectors. The thick dots in the upper left indicate when SNR $=8$ is accumulated. The red, green, and blue curves are the respective LIGO Voyager designs while the dashed line is aLIGO.

frequency sensitivity to accumulate SNR in the early part of the inspiral. We characterize early-warning detection with $t_{\text {early }}$, the time before coalescence at which a once-a-year event will accumulate SNR of 8 (optimally oriented binary, single detector). The SNR and sky localization accuracy as functions of time before merger are depicted in Fig. 6. Cosmological volume corrections have been taken into account, but the NS-NS merger rate is held constant at a fiducial value.

3.1.2. Intermediate Mass Black Holes The evidence for the existence of intermediate-mass black holes (IMBHs) in the $10^{2}-10^{4} \mathrm{M}_{\odot}$ mass range is still inconclusive at present. Attempts to look for electromagnetic signatures are hampered by the small dynamical footprint of low-mass IMBHs and the difficulty of associating phenomena such as ultraluminous $\mathrm{x}$-ray sources specifically with IMBHs (see [58] for a review). On the other hand, a handful of promising sources have been observed (e.g. [59]), and multiple formation scenarios have been proposed - though none without problems (see the introduction of [60] for a brief review). Thus, GW observations of compact objects in this mass range, which would be enabled by a future detector with good low-frequency sensitivity, could yield the first definitive proof of IMBH existence at the low end of the IMBH mass range [61]. Such measurements could also answer outstanding questions about the dynamics of globular clusters and about the formation history of today's massive black holes [62].

In general, three types of GW signatures of binary coalescences involving IMBHs can be detected by upgraded detectors:

- Intermediate-mass-ratio inspirals: If a reasonable fraction of globular clusters host IMBHs in the $100-1000 \mathrm{M}_{\odot}$ range, there is a good possibility of being able to detect intermediate-mass-ratio inspirals (IMRIs) of stellar-mass compact objects (NSs or BHs) into IMBHs. The dominant mechanism is likely to be the successive hardening of a binary involving an IMBH and a compact object by three-body interactions, until it can merge through GW radiation reaction [63-65]. Although IMBH occupation fractions in globular clusters are highly uncertain, we could assume that $10 \%$ of globular clusters host an IMBH in a suitable mass range. Globular clusters have a comoving density of $0.3 \mathrm{Mpc}^{-3}$. We use the fiducial values of $0.5 \mathrm{M}_{\odot}$ for interloper mass in 3-body interactions, $10^{5.5} \mathrm{pc}^{-3}$ for the stellar density and $10 \mathrm{~km} \mathrm{~s}^{-1}$ for the velocity dispersion. 
The merger rate depends on the component masses; it is $1 / T_{\mathrm{m}}$, where the merger time scale is

$$
T_{\mathrm{m}} \approx 3 \times 10^{8}\left(\frac{m}{M_{\odot}}\right)^{-0.2}\left(\frac{M}{100 M_{\odot}}\right)^{-0.4} \mathrm{yr},
$$

$M$ is the IMBH mass, and $m$ is the inspiraling compact object mass. We can assume that $M$ is distributed from $100-1000 \mathrm{M}_{\odot}$ with $p(M) \propto M^{-2}$, and $m$ is uniformly distributed from $1.2-12 \mathrm{M}_{\odot}$.

- IMBH binaries in stellar clusters: If a young dense stellar cluster has a sufficiently high binary fraction, and the deep core collapse timescale is sufficiently short, an IMBHIMBH binary could form via the collisional runaway scenario [66]. IMBH binaries could also form through the collision of two globular clusters, each containing an IMBH [67]. Estimates of the rates and mass distributions of these processes is highly uncertain, but a plausible framework for doing so is provided in Section 3.2 of [62].

- Low-mass MBH seeds: According to hierarchical models of massive black hole formation [68], today's massive black holes are the product of multiple mergers and accretion episodes, starting with light seeds of $\sim 100$ or a few hundred solar masses, possibly arising from the direct collapse of population III stars. If so, it may be possible to directly detect the first mergers of these low-mass seeds at redshifts of 10 or 15 , thereby testing these predictions $[69,70]$.

3.1.3. Testing General Relativity. GWs from compact-binary mergers will provide a unique probe of strong-field dynamics [71, 72]. LIGO's first observations of GWs from binary black holes have already allowed made it possible perform the first tests of general relativity (GR) in the highly relativistic strong-field regime [73]. LIGO Voyager instruments will allow us to significantly improve the precision of such tests.

Speed of propagation of GWs from joint GW-EM observations: According to GR, GWs travel at the speed of light, $c$. In other theories, the speed $v_{g}$ of propagation of GWs could be different [74]. Coincident observation of electromagnetic (EM) and GW signals from astrophysical sources such as GRBs or core-collapse supernovae make it possible to measure the time-delay $\Delta t_{a}$ between the EM and GW signals, and thus to constrain the speed of GWs. For the case of a source located at a distance $D$,

$$
1-\frac{v_{g}}{c} \simeq \frac{c \Delta t}{D} ; \quad \Delta t=\Delta t_{a}-\left[(1+z) \Delta t_{s}\right]
$$

where $\Delta t_{s}$ is the time-delay between the GW and EM emissions at the source and $z$ the cosmological red shift.

The most promising astrophysical sources for this test are short-hard GRBs [56]. It can be seen from Eq.(1) that the sensitivity of this test is proportional to the distance to the source, and the best bound is provided by sources located at the horizon distance of the detector. The precise bound that we can place on $v_{g}$ depends on the the time delay $\Delta t_{s}$ at the source, which is currently uncertain. Hence we use the horizon distance at which a double neutron star inspiral $\left(m_{1}=m_{2}=1.4 M_{\odot}\right)$ can be detected with an optimal SNR of 8 as a figure of merit for this measurement.

Mass of the graviton from joint GW-EM observations: One particular scenario in which the speed of GWs $v_{g}$ could differ from $c$ is in the case of graviton having a non-zero rest mass. 
This is characterized by the dispersion relation $v_{g}^{2} / c^{2}=1-m_{g}^{2} c^{4} / E_{g}^{2}$, where $m_{g}$ is the rest mass and $E_{g} \equiv 2 \pi \hbar f_{\mathrm{GW}}$ the rest energy of the graviton with frequency $f_{\mathrm{GW}}, \hbar$ being the reduced Planck constant [75]. From this dispersion relation and using Eq.(1), a lower bound on the Compton wavelength $\lambda_{g}=2 \pi \hbar / m_{g} c$ (or, an upper bound on the mass $m_{g}$ of the graviton) can be inferred from joint GW-EM measurements:

$$
\lambda_{g} \gtrsim\left[\frac{D c}{2 \Delta t f_{\mathrm{GW}}^{2}}\right]^{1 / 2}
$$

It can be seen that the best bound is provided by distant sources. Here we also use the horizon distance to a double neutron star inspiral $\left(m_{1}=m_{2}=1.4 M_{\odot}\right)$ as a figure of merit for this measurement.

Mass of the graviton from $G W$ observations of CBCs: $\mathrm{CBC}$ observations also make it possible to estimate the mass of the graviton even in the absence of an EM counterpart. In the case of CBCs, the GW frequency sweeps from lower to higher frequencies. If the graviton is massive, different frequency components travel with different speeds, causing a distortion in the observed waveform [75]. In particular, the observed GW phase $\Psi(f)$ in the frequency domain will be deviated from the phase $\Psi_{\mathrm{GR}}(f)$ predicted by GR:

$$
\Psi(f)=\Psi_{\mathrm{GR}}(f)-\frac{\pi D}{\lambda_{g}^{2}(1+z)} f^{-1}
$$

where $\lambda_{g} \equiv h / m_{g} c$ is the Compton wavelength of the graviton. LIGO's first observation of a binary black hole system has provided one of the best lower bound on $\lambda_{g} \sim 10^{13} \mathrm{~km}$ [76]. Here we use the expected lower bound on $\lambda_{g}$ from the observation of a binary black hole system with parameters similar to the first LIGO event $\left(m_{1}=m_{2}=30 M_{\odot}\right.$, located at a distance of $500 \mathrm{Mpc}$ ) as the figure of merit. The bounds reported in Table 1 are computed using the Fisher matrix formalism, outlined in [77].

Decay of GWs during propagation: If GWs decay during propagation (apart from the expected $1 / r$ falloff; e.g. due to dissipation), distant sources would appear to be systematically dimmer. The detection of this requires a population of coincident GW+EM observations with redshift $z$ estimation (say, from the merger binary neutron stars). Then we could look for a systematic suppression of GW amplitude for higher- $z$ sources. The sensitivity of this test would be proportional to the distance traveled by the GWs. Assuming that the redshift can be accurately estimated for sources located at arbitrary distances, the relevant figure of merit for GW detectors is simply the horizon distance. We take the horizon distance (SNR of 8) to non-spinning binary neutron star inspirals with $m_{1}=m_{2}=1.4 M_{\odot}$ as the figure of merit for this test.

Parametrized deviations from post-Newtonian theory: Here we introduce parametrized deviations from GR in the inspiral waveforms computed using the post-Newtonian (PN) approxmation to GR, and examine our ability to constrain these deviations from the data. Consistency with their GR values is a null-hypothesis test of relativity [78-80].

The frequency domain phase of the PN waveforms can be written as:

$$
\Psi(f)=2 \pi f t_{0}+\phi_{0}+\sum_{k=0}^{7}\left(\psi_{k}+\psi_{k}^{\mathrm{L}} \ln f\right) f^{(k-5) / 3},
$$


where $\psi_{k}$ and $\psi_{k}^{\mathrm{L}}$ are the PN coefficients of the phase at order $k / 2 \mathrm{PN}$. We introduce deviations in the PN coefficients in the following way:

$$
\psi_{k} \rightarrow \psi_{k}+\Delta \chi_{k}, \quad \psi_{k}^{\mathrm{L}} \rightarrow \psi_{k}^{\mathrm{L}}+\Delta \chi_{k}^{\mathrm{L}},
$$

where $\Delta \chi_{k}$ and $\Delta \chi_{k}^{\mathrm{L}}$ are zero in GR. For a non-spinning binary, the waveform $h(f)=$ $A(f) \exp [i \Psi(f)]$ depends on the intrinsic binary parameters $m_{1}, m_{2}$, the extrinsic parameters $t_{0}, \phi_{0}, D$ and the deviation parameters $\Delta \chi_{k}, \Delta \chi_{k}^{\mathrm{L}}$. We deform the GR waveform by introducing one deviation parameter at a time and compute the expected constraint on this from the Cramér-Rao bound, from an archetypal binary neutron star system with $m_{1}=m_{2}=1.4 M_{\odot}$ located at a distance of $500 \mathrm{Mpc}$. The $1 \sigma$ errors in estimating the deviation parameter $\Delta \chi_{7}$ at 3.5PN order, as a fraction of the known value $\psi_{7}$ of the 3.5PN term in GR, are given in Table 1.

Detecting the tails: The backscattering of gravitational waves emitted by a binary are by the binary's own gravitational field[81, 82] are known as tails. In a post-Newtonian expansion, this effect appears first in the 1.5 PN phasing coefficient. The figure of merit will be our estimation error for this coefficient (that is, $\Delta \chi_{3}$ as defined in the previous section) from a double neutron star system $\left(m_{1}=m_{2}=1.4 M_{\odot}\right)$ located at $500 \mathrm{Mpc}$.

Detecting the memory effect: The memory wave can be viewed as the gravitational effect of the stress-energy carried by previously emitted waves (Christodoulou, or nonlinear memory) with a contribution from the final momentum distribution of the binary (linear memory, e.g., due to kick) [83-86]. The ramping up of the memory wave may be a measurable [87-89] test of GR. Let us provide a simple estimate of this effect, by writing

$$
h^{\mathrm{mem}}(t) \propto \frac{R^{2}}{M} \int_{-\infty}^{t} \dot{h}^{2}\left(t^{\prime}\right) d t^{\prime}
$$

Here $h$ is the leading gravitational waveform, while $h^{\mathrm{mem}}$ is the nonlinear memory effect generated by the stress-energy associated with $h$. We note that $h^{\mathrm{mem}}$ is mostly a slowly increasing function of time, except during the merger process. We can use the SNR for $h^{\mathrm{mem}}$ as the FOM for detecting the memory effect. We choose a fiducial BBH of $(30+30) \mathrm{M}_{\odot}$ at $500 \mathrm{Mpc}$ as representative for this FOM.

Testing the no-hair theorem with inspirals According to the no-hair theorem of General Relativity, the spacetime around a singularity fully enclosed by an event horizon, with no closed timelike curves outside the horizon, and subject to several additional conditions, must be described by the Kerr metric. In particular, the full structure of the spacetime can be described by a multipole moment decomposition with only two free parameters, mass and spin, and all higher-order moments given by the mass and spin.

Ryan proved that the full multipole moment structure of the spacetime can in principle be measured through observations of gravitational-wave emission [90]. Therefore, gravitational waves can be used to test deviations from the null hypothesis that massive compact objects are black holes by checking for consistency between higher-order multipole moments and their values as predicted by the Kerr metric. The mass quadrupole moment is the first measurable term beyond the mass and spin, entering the post-Newtonian expansion at the second postNewtonian order in the phase. Therefore, its measurement is likely to be the focus of no-hair theorem tests, though other tests (e.g., through consistency of ringdown modes [e.g., 91, and see below]) are possible. 
Intermediate-mass-ratio inspirals (IMRIs) of low-mass compact objects into intermediate mass black holes provide a particularly promising tool for constraining the mass quadrupole moment and null hypothesis deviations [92, 93]. Given the uncertainty in existing waveform families in the intermediate-mass-ratio regime [e.g., 94], the exact precision of the measurement is at present compromised by systematic waveform uncertainty. Therefore, we will use the SNR of an intermediate-mass-ratio inspiral of a 1.4 solar mass neutron star into a $100 \mathrm{M}_{\odot}$ black hole at a luminosity distance of $1 \mathrm{Gpc}$ (consistent with an astrophysically plausible rate of such inspirals in globular clusters [63]) as a proxy for the detectability of IMRIs and the measurability of the mass quadrupole moment.

Testing the No-Hair theorem with Ringdowns GW signals from the ringdown phase of a BBH coalescence are expected to be dominated by a spectrum of quasi-normal modes (QNMs). According to the no-hair theorem, the frequencies $\omega_{\ell m}^{\mathrm{GR}}$ and damping times $\tau_{\ell m}^{\mathrm{GR}}$ of these QNMs are unique functions of the mass $M_{f}$ and spin $a_{f}$ of the final Kerr black hole [95]. Thus, in principle, the mass and spin of the final black hole can be extracted from different QNMs, which have to be consistent with each other. In practice, one can introduce parameters $\Delta \omega_{\ell m}$ and $\Delta \tau_{\ell m}$ that describe deviations from the GR prediction of the frequencies and damping times of the QNMs

$$
\omega_{\ell m}=\omega_{\ell m}^{\mathrm{GR}}\left(M_{f}, a_{f}\right)\left(1+\Delta \omega_{\ell m}\right), \quad \tau_{\ell m}=\tau_{\ell m}^{\mathrm{GR}}\left(M_{f}, a_{f}\right)\left(1+\Delta \tau_{\ell m}\right) .
$$

and constrain those deviations [91]. We use the SNR of the ringdown phase of the expected signal from a BBH system with $m_{1}=m_{2}=30 \mathrm{M}_{\odot}$, located at a distance of $500 \mathrm{Mpc}$ as a simple FOM for our ability to constrain these deviation parameters.

Unaccounted loss of energy and angular momentum: If a $\mathrm{GW}$ signal from a $\mathrm{BBH}$ coalescence is observed with sufficient SNR, the masses and spins of the black holes can be estimated from just the inspiral part of the signal. Using these estimates of the initial parameters of the binary, the mass and spin of the final black hole can be uniquely predicted by making use of numerical relativity simulations. In addition, the mass and spin of the final black hole can be independently estimated from the ringdown part of the signal [96, 97]. Any inconsistency between these two estimates will point to an unaccounted loss in the energy / angular momentum from the system.

This consistency test requires binaries with the right masses and spins such that the inspiral, merger and ringdown parts of the signal are all observed with sufficiently large SNRs. As a simple FOM, we use the SNR of a non-spinning binary with $m_{1}=m_{2}=30 M_{\odot}$, located at $500 \mathrm{Mpc}$.

3.1.4. Dense Matter Equation of State from the Tidal Deformation of Neutron Stars The inspiral and merger of $\mathrm{BH} / \mathrm{NS}$ or NS/NS binaries can provide a wealth of information about the NS Equation of State (EOS). This may come about through observing the phase evolution of a NS/NS or $\mathrm{BH} / \mathrm{NS}$ in the late inspiral to merger, the pulsations of a hypermassive NS (or newly-born stable NS), which may form during a NS/NS merger, and the frequency of tidal disruption of the NS in a BH/NS inspiral; see [98] for inference on the EOS from the gravitational-wave signal of the NS/NS merger GW170817.

For most of the NS/NS or BH/NS inspiral, NSs are well approximated as point particles; detections and rate estimates can be made with templates that ignore finite size effects. However, as the size of the orbit becomes comparable to the size of the neutron stars, the EoS will begin to modify the phase evolution as tidal effects deform the neutron stars. These 


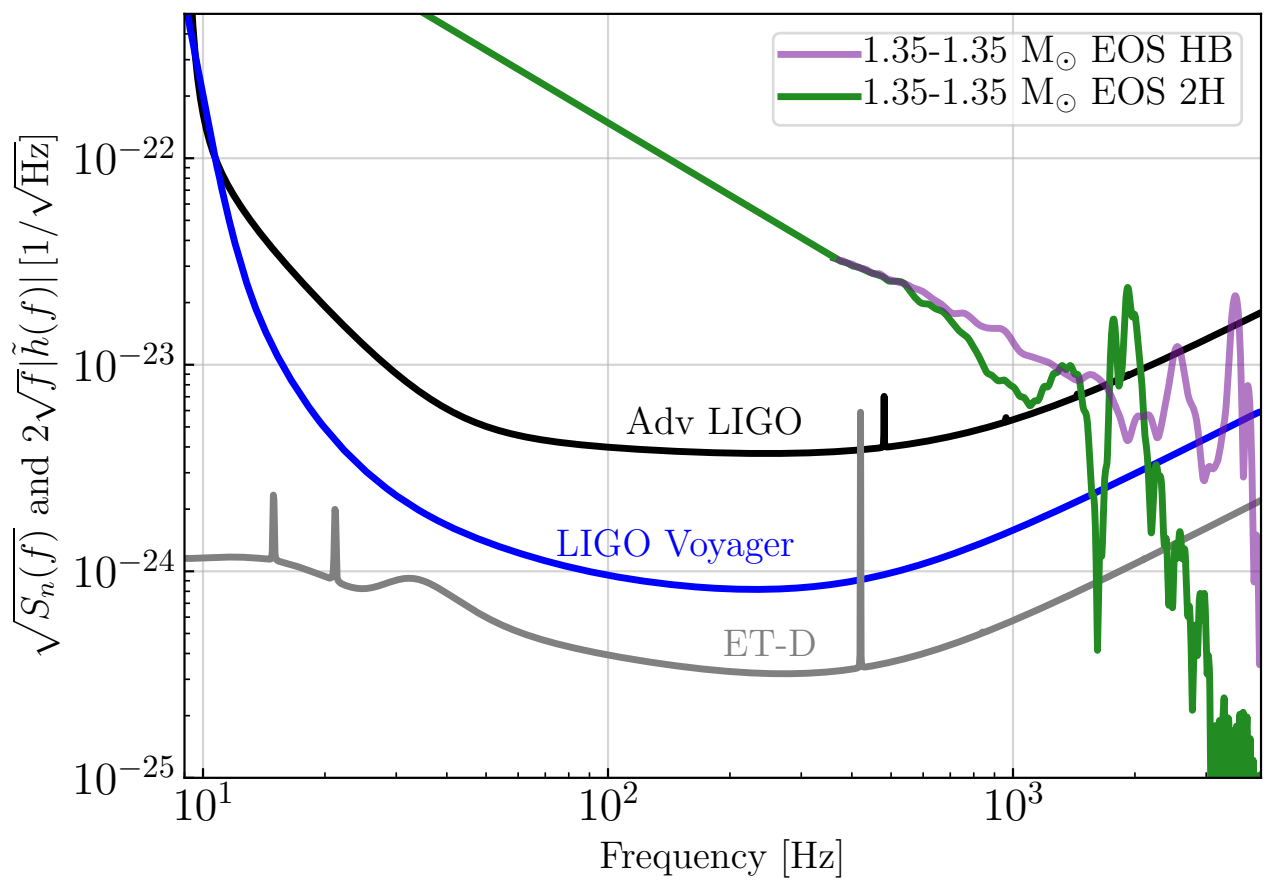

Figure 7: The amplitudes of NS/NS waveforms, compared to noise spectra of various GW detectors, showing the frequency range where various EoS effects may be seen. Hybrid waveforms for merging binary neutron stars with two different EoS from [99] are shown. EoS HB produces a neutron-star radius of $11.6 \mathrm{~km}$ and EoS $2 \mathrm{H}$ a radius of $15.2 \mathrm{~km}$. Both waveforms are from $1.35-1.35 \mathrm{M}_{\odot}$ binaries at an effective distance of $100 \mathrm{Mpc}$. Differences in waveform amplitudes due to strong tidal interactions are seen before the merger (up to $\sim 1000 \mathrm{~Hz}$ for EoS $2 \mathrm{H}$, and up to $\sim 2000 \mathrm{~Hz}$ for EoS HB.) The effects of the EoS on the phase evolution are not visible in the amplitudes below $\sim 500 \mathrm{~Hz}$ but would be measurable in Advanced LIGO for extreme EoS like 2H [100]. Post-merger oscillations of a hypermassive neutron star formed by the merger produce peaks in the spectrum near $2000 \mathrm{~Hz}$ for EoS $2 \mathrm{H}$, and near $3500 \mathrm{~Hz}$ for EoS HB.

modifications in the high-frequency portions of the waveforms are shown in Figure 7 for two representative equations of state.

The leading order effect of the EoS on the GWs is characterized by a parameter that relates the size of the quadrupole deformation induced in the star to the strength of the external tidal field. A dimensionless form of this parameter is

$$
\Lambda=\frac{2}{3} k_{2}\left(\frac{R}{M}\right)^{5},
$$

which depends on the Love number $k_{2}$, the radius of the star $R$, and the mass $M$ of the star. Both $k_{2}$ and $R$ are determined by the EoS. Tidal effects contribute to the waveform formally at 5 th and higher post-Newtonian (PN) orders [101, 102]. The PN model will break down at high frequency, as the stars interact more strongly, and eventually as the stars collide at a frequency which depends on the EoS. However, the magnitude of the waveform effects predicted in PN models is approximately equal to the magnitude of effects seen in both EOB calculations [103] and estimates from hybrid waveforms using numerical simulations [104]. In this paper, we therefore use the leading order tidal contribution in TaylorF2 post-Newtonian models to estimate the measurability of EoS effects. In practice, a more accurate waveform model will be required to measure this parameter without significant systematic errors $[98,105,106]$. 


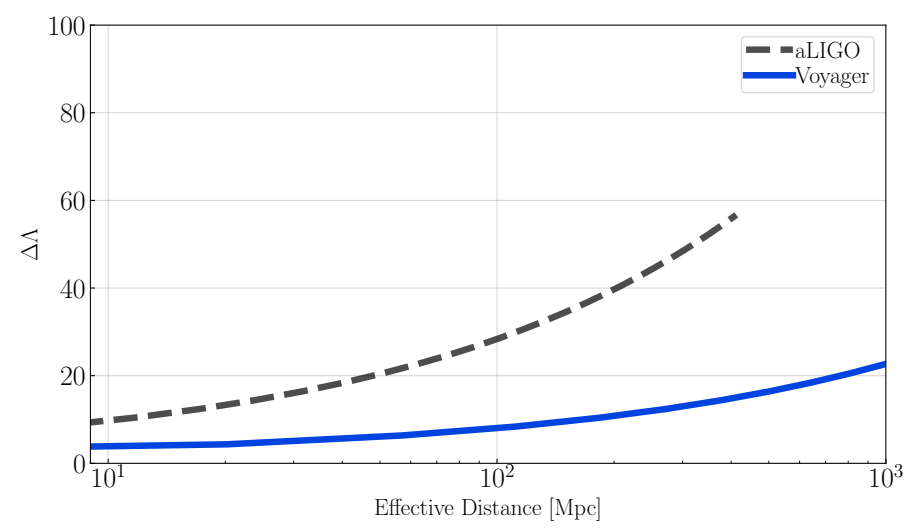

Figure 8: Tidal deformability figure of merit. $\Lambda$ is the parameter characterizing the tidal deformability of various equations of state. We show the maximum effective distance at which a given difference in $\Lambda$ is distinguishable. This figure considers a NS-NS, $1.4-1.4 \mathrm{M}_{\odot}$ binary, with waveforms generated using Taylor T4, with tidal terms parametrized by $\Lambda$. Note that to distinguish between the SLY and AP4 equations of state, for example, one would need to find $\Delta \Lambda \approx 50$.

The figure of merit for neutron star tidal deformability is related to how well we can differentiate between different values of $\Lambda$ (and hence different equations of state) for various noise curves. We estimate how well we can differentiate between two waveforms using the distinguishability criteria $\delta h$, as discussed in [104]. We find $\delta h$ for a given system at $100 \mathrm{Mpc}$, and then compute $100 \mathrm{Mpc} \times \delta h$ to find the effective distance at which $\delta h=1$ (the minimum distance for distinguishability).

We consider a $1.4-1.4 \mathrm{M}_{\odot}$ NS-NS binary, and find how well we can recover a given $\Lambda$ by computing overlaps of Taylor T4 waveforms. For our figure of merit, we consider the maximum effective distance at which the SLY [107] and AP4 [108] equations of state can be distinguished, where $\Lambda_{\mathrm{SLY}}=323$ and $\Lambda_{\mathrm{AP} 4}=270$ for the given binary system. The accuracy in the recovery of the tidal deformability parameter $\Lambda$ is plotted in Figure 8 . For NS/BH binaries, we consider the maximum effective distance where these two equations of state would be distinguishable in a $1.4-10.0 \mathrm{M}_{\odot}$ binary. The Jacobian quantities using this FOM are then computed by the same prescription as in the rest of this study.

3.1.5. Measurement of the Cosmological Expansion In the era of precision cosmology, measurements of cosmological parameters are based primarily on two types of observations: standard candles and large-scale structure. Standard candle measurements rely on measuring data points in the distance-redshift Hubble diagram by considering sources with a known intrinsic luminosity, such as type IA supernovae, allowing the distance to be extracted from their apparent luminosity. Such observations famously led to the discovery of the acceleration of the cosmological expansion of the Universe [109]. Together with measurements of large-scale structure, and particularly of the cosmic microwave background radiation, these observations have led to modern concordance cosmology: a flat Universe, with a Hubble constant of approximately $70 \mathrm{~km} / \mathrm{s} / \mathrm{Mpc}$, dominated by dark energy $\left(\Omega_{\Lambda} \approx 0.7\right)$ and cold dark matter, with a dark energy equation of state $(p=w \rho)$ parameter $w=-1$ within $\sim 15 \%$ [110]. Despite the success of these observations, they may suffer from systematic errors, such as any redshift dependence in type IA SNe instrinsic luminosity. There is also mild contention between the WMAP and Planck data regarding the value of the Hubble 
constant [111]. Gravitational waves may therefore provide a compelling alternative measurement of cosmography, since they are sensitive to different sources and subject to different systematics $[112,113]$. The promise of gravitational-wave cosmology was pointed out by Schutz [114]. Although gravitational-wave observations of binary inspirals can be used to directly measure the distance to the source, making them standard sirens [115], the redshift is generally degenerate with the source mass, since the mass provides the only timescale in the evolution of the binary.

Several possibilities exist for breaking this degeneracy and measuring the binary's redshift. If the binary is associated with an electromagnetic counterpart that allows accurate localization to a unique host galaxy, the redshift can be measured directly. This was done for GW170817, with a resulting measurement of the Hubble constant at the 15\% level [116]. Otherwise, in the presence of a galaxy catalog and under the assumption that binaries merge in galaxies, a statistical association is possible $[114,117]$. If one of the binary components is a neutron star, matter effects (e.g., tides) provide an alternative scale in the problem - the physical size of the neutron star - making it possible to break the mass-redshift degeneracy [118, 119]. Finally, if the intrinsic distribution of the source masses is sufficiently narrow, the degeneracy can again be broken [120].

Although all of the above approaches are promising, in this study we will use the accuracy of the measurement of the Hubble constant based on the intrinsically narrow distribution of neutron star masses in binary neutron star systems as the figure of merit. A useful scaling law for the fractional Hubble constant measurement accuracy [120] is:

$$
\frac{\delta H}{H} \approx \frac{1}{\sqrt{N}}\left[\frac{\sigma_{\mathcal{M}}}{z \mathcal{M}}+\frac{\delta d_{L}}{d_{L}}\right],
$$

where the fractional spread in the binary neutron star chirp mass is $\sigma_{\mathcal{M}} / \mathcal{M} \approx$ $\left(0.06 M_{\odot}\right) /\left(1.2 M_{\odot}\right)=0.05[121], z$ is the maximum redshift at which a neutron star binary can be detected, $N$ is the total number of detections, and $\delta d_{L} / d_{L} \approx 0.3$ is the fractional uncertainty on the distance measurement for the most distant source [122].

\subsection{Astrophysics with Stellar Collapse}

The core collapse of massive stars has long been considered an interesting source of GWs [123]. While the intricacies of the core-collapse supernova (CCSN) explosion mechanism are not well understood, state-of-the-art 3D simulations (see, e.g., [124-145]) suggest that rapid rotation, turbulent convection, and instabilities of the stalled accretion shock play important roles in re-energising the shock and aiding stellar explosion. GW emission from the initial core collapse and subsequent explosion is strongly influenced by the physical processes driving the explosion. For this reason, GW observations can be used to directly probe the CCSN central engine and gain insight into the explosion mechanism [146, 147].

The angular momentum and degree of differential rotation of the precollapse stellar core are thought to strongly influence the dynamics of the initial collapse, the subsequent explosion, and the compact remnant formed (see, e.g. [148-151]). Observations of the evolving pulsar population suggest a broad distribution of moderately rotating NSs at birth with initial spin periods around $10-100 \mathrm{~ms}$ [152-154]. Wave-driven angular momentum transport in massive stars during the late stages of shell burning may strongly impact the pre-collapse rotation rate, predicting a distribution of initial NS periods consistent with observations [155]. Binary interactions are also expected to have a marked effect on the rotation of massive stars [156158]. 
For stellar cores with pre-collapse periods exceeding a few tens of seconds, delayed revival of the stalled shock is thought to be driven by the neutrino mechanism. In this scenario, some small fraction of the binding energy released in neutrinos is absorbed in a layer between the stalled shock and proto-NS. Increased pressure behind the shock from neutrino heating and multi-dimensional hydrodynamic instabilities drive it outwards and aid explosion.

State-of-the-art 3D simulations suggest that turbulent convection and the standing accretionshock instability (SASI) are expected to dominate the explosion dynamics [124, 127$131,159,160]$. Extensive research on the GW signature from slowly rotating core collapse has been done in 2D (see, e.g.,[160, 161], for recent studies) and 3D (see, e.g., [125, 139, 140, 159, 162] for recent studies).

Proto-neutron star (PNS) oscillation modes can source appreciable GW emission from $(100-200) \mathrm{ms}$ after core bounce, following a short quiescent period, for up to $\sim 1 \mathrm{~s}$. The GW frequency naturally follows the dominant PNS surface g-mode frequency, increasing linearly with time from $\sim(100-200) \mathrm{Hz}$ to over $1 \mathrm{kHz}$ as the PNS evolves [160-164]. Strong fluid downflows associated with the SASI can modify the accretion rate at the PNS, inducing quadrupolar oscillations at $\sim(100-200) \mathrm{Hz}$ at later times [125, 163, 164].

GW memory, a non-oscillatory contribution to the GW amplitude at leading quadrupole order (see, e.g. [84, 165]), may be created by anisotropic neutrino emission [160, 162, 166-173] and aspherical explosive outflows (e.g., in jets or if the shock acceleration is not spherically symmetric) of matter and magnetic stresses [168, 174-176]. For anisotropic neutrino emission, the GW memory effect causes emission at less than $10 \mathrm{~Hz}[160,162,168,170,171]$. Rapidly rotating stars, which are expected to make up $\sim 1-10 \%$ of the massive star population [177-179], could explode via a bipolar magnetohydrodynamic explosion leading to large explosion asymmetries and relativistic outflows [126, 132, 145, 180-185]. The inner cores in rotating progenitor stars become centrifugally deformed in the late stages of collapse. This results in a large quadrupole moment, which changes rapidly at core bounce, leading to a strong and pronounced peak in the GW signal that is followed by ring-down oscillations of the PNS [182, 185-187].

PNSs with strongly differentially rotating profiles are often subject to a rotational shear instability that drives the development of nonaxisymmetric dynamics in the PNS core [168, 188-191]. Also known as the co-rotational (or low $T /|W|$ ) instability, typical GW energy emitted can be as high as $10^{-7} M_{\odot} c^{2}$.

A number of energetic SN explosions have been seen in coincidence with nearby long gammaray bursts (GRBs), providing an observationally robust connection between long GRBs and stellar collapse [192, 193]. The central engine in a long GRB is thought to be either a nascent black hole surrounded by a fallback accretion disk (a collapsar [194-196]) or millisecond proto-magnetar [197, 198]. In systems with accretion disks from fallback material, various instabilities may develop and lead to GW emission (e.g., [199-201]). Classical dynamical instabilities are unlikely to occur in regular core collapse events, but may be relevant in extreme cases that lead to long GRBs and/or black hole formation [168, 202-206].

$\mathrm{BH}$ formation as a consequence of fallback accretion onto the PNS is thought to be the formation channel for most stellar BHs [204, 207]. The timescale on which this occurs is dependent on the accretion rate (directly influenced by the properties of the progenitor star), the angular momentum of the PNS, and the nuclear matter equation of state (EOS). In most systems, this happens $\sim 0.5-3 \mathrm{~s}$ after core bounce, and is characterised by a short GW burst with a broad spectrum peaking at $2.5-3.5 \mathrm{kHz}$ [204, 208, 209].

Rapid rotation is also expected to be present in massive accreting white dwarfs and in the cores of white dwarf merger remnants (e.g., [210]). Such massive degenerate cores are expected to collapse to neutron stars rather than explode as thermonuclear supernovae. 
Such "accretion-induced collapse" (AIC) events are expected to give off a strong burst of gravitational waves $[168,211]$.

3.2.1. Detection Prospects. The galactic rate of core collapse events is low and estimates vary from $\sim 1$ in 40 years to 1 per century [212-214]; including the Large and Small Magellanic Clouds and the Andromeda galaxy (M31) roughly doubles the rate [212]. A significant increase in the event rate occurs only outside of the Local Group of Galaxies with the M81 group of galaxies at $\sim 3-5 \mathrm{Mpc}[215,216]$. There, $\sim 0.5$ core-collapse supernovae are discovered per year, suggesting a rate of around 1 event per year, when assuming that $\sim 50 \%$ of the events remain undiscovered due to obscuration or weak/absent EM emission. The integrated event rate out to $10 \mathrm{Mpc}$ is likely around $\gtrsim 2$ events per year $[215,216]$.

While the search for CCSNe with the first generation of ground-based GW detectors yielded no observations, constraints on energy emitted in GWs by CCSNe were made for the first time [217]. With the second-generation instruments, we don't expect to see the typical CCSN beyond a few kpc for slowly rotating progenitors, while rapidly rotating progenitors might yield GW emission observable throughout the galaxy and Magellanic clouds [218-220].

3.2.2. Probing Core-Collapse Supernova Physics. The physics that may be learned from a detection of GWs from stellar collapse goes far beyond constraining GW emission and explosion mechanisms. CCSNe and related phenomena are cosmic laboratories for highenergy-density gravitational, plasma, nuclear, and particle physics. In particular, it may be possible to extract information on the nuclear EOS directly from GW observations [125, 163, 170, 185, 221, 222]. Electromagnetic (EM) observations can tell us little directly about the distribution of rotation rates of pre-collapse iron cores, such that existing constraints come primarily from observations of the resultant young compact objects. For rapidly rotating pre-collapse cores, GW observations can be used to directly infer the angular momentum distribution [187, 223, 224], aided by multimessenger observations with neutrinos [225]. Coincident GW and neutrino observations will be of extreme importance if the next Galactic core collapse event leads to black hole formation (without electromagnetic display). MeVenergy neutrinos from any Galactic or nearby extragalatic core collapse event will be observed by current and future neutrino detectors (e.g., [226-230]).

In the absence of a more specific FOM covering GWs from both slowly and rapidly rotating core collapse, we consider the signal SNR as the astrophysical FOM. LIGO Voyager, in the baseline design considered in this report, yields SNRs for core-collapse supernova waveforms (see Table 4 and Section 3.2) that are a factor of 4-5 greater than aLIGO, which means that robust core-collapse supernova model selection may be possible out to distances of $\sim 8-$ $10 \mathrm{kpc}$, providing coverage virtually throughout the Galaxy. There will likely be at most one core collapse event in the Milky Way in the lifetime of LIGO, so extending our reach throughout the Galaxy is crucial.

According to the current understanding of core-collapse supernova theory [231], the most likely and most robust GW emission mechanism is turbulent neutrino-driven convection in the context of the "neutrino mechanism" $[168,173]$. This leads to broadband GW emission with most power at $100-1000 \mathrm{~Hz}$. Table 4 shows that most improvement above the baseline LIGO Voyager design will come from reducing the shot noise either through more squeezing or more laser power. Note that the vast majority of stellar collapse events lead to standardenergy type-II supernovae and are unlikely to be strong GW emitters. Even LIGO Voyager will not be able to observe such events to distances greater than $\sim 100 \mathrm{kpc}$, which covers the Milky Way, and the Magellanic Clouds. 


\begin{tabular}{|c|c|c|c|c|c|c|c|c|c|c|}
\hline Waveform Type & $\begin{array}{c}\text { aLIGO SNR } \\
\text { @ } 10 \mathrm{kpc}\end{array}$ & $\begin{array}{l}\text { LIGO Voyager SNR } \\
\text { baseline @ } 10 \mathrm{kpc}\end{array}$ & $\mathrm{NN}$ & SEI & sus & SPOT/CTN & SQZ & POW & FCL & MASS \\
\hline \multicolumn{11}{|l|}{$v$ GW Memory } \\
\hline $\begin{array}{l}\text { B12-WH07-v (2D) }[160] \\
\text { B20-WH07-v (2D) [160] }\end{array}$ & $\begin{array}{l}19.70 \\
16.54\end{array}$ & $\begin{array}{l}75.91 \\
63.73\end{array}$ & $\begin{array}{l}4.22 \times 10^{-3} \\
4.18 \times 10^{-3}\end{array}$ & $3.93 \times 10^{-4}$ & $\begin{array}{l}1.00 \times 10^{-2} \\
100 e-02 \times 10^{-2}\end{array}$ & $3.14 \times 10^{-1}$ & $\begin{array}{l}-2.18 \times 10^{-2} \\
-2.19 \times 10^{-2}\end{array}$ & $3.40 \times 10^{-1}$ & $2.20 \times 10^{-1}$ & $\begin{array}{l}-1.86 \times 10^{-1} \\
-1.86 \times 10^{-1}\end{array}$ \\
\hline \multicolumn{11}{|l|}{ Rapidly Rot. Core Collapse } \\
\hline $\mathrm{R} \mathrm{E} 1 \mathrm{CA} \mathrm{L}_{\mathrm{L}}(3 \mathrm{D})[145]$ & 1.46 & 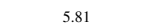 & $1.37 \times 10^{-6}$ & $5.67 \times 10^{-8}$ & $5.70 \times 10^{-6}$ & $1.29 \times 10^{-1}$ & $6.22 \times 10^{-1}$ & $3.39 \times 10^{-1}$ & $-1.62 \times 10^{-3}$ & $-6.65 \times 10^{-3}$ \\
\hline R3E1AC $(3 D)[145]$ & 74.38 & 287.87 & $2.43 \times 10^{-7}$ & $1.14 \times 10^{-8}$ & $1.09 \times 10^{-6}$ & $7.49 \times 10^{-2}$ & $7.03 \times 10^{-1}$ & $3.81 \times 10^{-1}$ & $-8.73 \times 10^{-4}$ & $-1.84 \times 10^{-3}$ \\
\hline $\mathrm{R} 4 \mathrm{E} \mathrm{FC}_{\mathrm{L}}$ (3D) [145] & 77.12 & 287.53 & $4.42 \times 10^{-7}$ & $1.40 \times 10^{-8}$ & $2.22 \times 10^{-6}$ & $5.99 \times 10^{-2}$ & $7.38 \times 10^{-1}$ & $3.99 \times 10^{-1}$ & $-7.69 \times 10^{-4}$ & $-2.65 \times 10^{-3}$ \\
\hline R3 (3D) [182] & 30.48 & 123.72 & $1.98 \times 10^{-5}$ & $4.95 \times 10^{-7}$ & $8.17 \times 10^{-5}$ & $2.21 \times 10^{-1}$ & $5.04 \times 10^{-1}$ & $2.82 \times 10^{-1}$ & $-4.10 \times 10^{-3}$ & $-4.28 \times 10^{-2}$ \\
\hline \multicolumn{11}{|l|}{ Convec } \\
\hline L15-3 & 3. & 15.39 & -4 & $\times 10^{-5}$ & $10^{-3}$ & $0^{-1}$ & $3.58 \times 10^{-1}$ & $2.14 \times 10^{-1}$ & $-8.59 \times 10^{-3}$ & $-9.46 \times 10^{-2}$ \\
\hline D) $[234]$ & 3.4 & 13.70 & 1.2 & 4.30 & & & 5.84 & $10^{-1}$ & -2.3 & $10^{-2}$ \\
\hline $\mathrm{s} 20 \mathrm{~s}(3 \mathrm{I}$ & 5.5 & 21.9 & $1.85 \times 10^{-4}$ & $9.08 \times 10^{-6}$ & $6.14 \times$ & & 5.52 & 3.09 & -5.15 & $-5.31 \times 10^{-2}$ \\
\hline TM1 (3D) [125] & 8.43 & 32.14 & $6.94 \times 10^{-7}$ & $1.33 \times 10^{-8}$ & $2.16 \times 10^{-5}$ & 1.33 & $6.39 \times 10^{-1}$ & $3.51 \times 10^{-1}$ & $-2.67 \times 10^{-3}$ & $-3.31 \times 10^{-2}$ \\
\hline $\mathrm{SFHx}$ (3D) [125] & 12.48 & 47.75 & $7.20 \times 10^{-7}$ & $1.76 \times 10^{-8}$ & $2.75 \times 10^{-5}$ & $1.79 \times 10^{-1}$ & $5.83 \times 10^{-1}$ & $3.24 \times 10^{-1}$ & $-3.87 \times 10^{-3}$ & $-5.21 \times 10^{-2}$ \\
\hline \multicolumn{11}{|l|}{ BH Formation in Colla } \\
\hline $\begin{array}{l}\text { u75rot1 (3D) [209] } \\
\text { u75rot2 (3D) [209] }\end{array}$ & $\begin{array}{l}11.77 \\
30.91\end{array}$ & $\begin{array}{c}43.84 \\
114.84\end{array}$ & $\begin{array}{l}1.81 \times 10^{-7} \\
5.70 \times 10^{-7}\end{array}$ & $\begin{array}{l}3.55 \times 10^{-9} \\
1.32 \times 10^{-8}\end{array}$ & $\begin{array}{l}1.76 \times 10^{-6} \\
2.98 \times 10^{-6}\end{array}$ & $\begin{array}{l}7.33 \times 10^{-2} \\
7.08 \times 10^{-2}\end{array}$ & $\begin{array}{l}7.18 \times 10^{-1} \\
7.22 \times 10^{-1}\end{array}$ & $\begin{array}{l}3.89 \times 10^{-1} \\
3.91 \times 10^{-1}\end{array}$ & $\begin{array}{l}-9.13 \times 10^{-1} \\
-8.76 \times 10^{-4}\end{array}$ & $-2.82 \times 10^{-3}$ \\
\hline
\end{tabular}

Table 4: Jacobian of Science Return as a function of interferometer upgrade technology for various stellar collapse waveform families introduced in Section 3.2. The baseline FOM is the angle-averaged SNR $<\rho>$.

A number of theoretical models (Section 3.2) predict strong GW emission connected with hyper-energetic core-collapse supernovae and/or long-duration GRBs. A potential candidate emission process relies on long lasting non-axisymmetric bar-like deformations of an extremely rapidly spinning PNS (or "protomagnetar") due to a rotational instability $[168,202,206,232]$. The GW emission is expected to be narrow-band and at high frequency $(\sim 400-2000 \mathrm{~Hz})$ and Table 4 lists results for a range of potential waveforms, generated using the ad-hoc bar model of [232]. Even the weakest signal considered here may be detectable by aLIGO at a distance of a few hundred kpc. LIGO Voyager in its baseline configuration could be able to detect this signal out to $5-10 \mathrm{Mpc}$. At this distance, $\gtrsim 2$ core collapse events occur per year. It would thus be possible to put strong constraint on the presence of such strong emission models.

3.2.3. Gravitational-Wave Memory in Stellar Collapse in the Milky Way GW memory may be left behind by most stellar collapse events, even those that do not result in an explosion. The typical growth timescale of the memory is of order $\gtrsim 0.1 \mathrm{~s}$, which makes it the only known low-frequency GW emission process in stellar collapse. Detecting the GW memory from a galactic event with aLIGO may be a difficult task even if the full projected low-frequency sensitivity is reached, but the baseline LIGO Voyager design would allow detection. Searches for GW memory would most benefit from improvements of the coating thermal noise or the arm cavity spot size (see Table 4).

\subsection{Astrophysics with Neutron Stars}

3.3.1. Bursting Magnetars, Glitching Pulsars. Magnetars are neutron stars powered by extreme magnetic fields $\left(\sim 10^{15} \mathrm{G}\right)$ [235]. They are thought to be the progenitors for the soft gamma repeaters (SGRs) and the anomalous X-ray pulsars (AXPs), compact X-ray sources which give steeply rising bursts of soft gamma rays typically lasting less than a second and with total isotropic burst energies rarely exceeding $10^{42} \mathrm{erg}$ (for a review see [236]). Only a few dozen SGRs and AXPs are known. Three extraordinarily giant flares have been observed in $~ 30$ years from magnetars in our Galaxy and the Large Magellanic Cloud with observed energies of between $\sim 1.2 \times 10^{44} d_{55}^{2}$ erg [237] and $\sim 5 \times 10^{46} d_{15}^{2}$ erg [238] where $d_{n}=d /(n \mathrm{kpc})$. Some short gamma ray bursts (GRBs) might be extragalactic giant flares. GRB 070201 might have been a giant flare located in the Andromeda galaxy with an isotropic energy of $1.5 \times 10^{45} \mathrm{erg}[239,240]$; and GRB 051103 might have been a giant flare in M81 with an energy of $7.5 \times 10^{46} \mathrm{erg}[241]$. 
GW signals from magnetars would allow us to probe NS physics and structure. However, it is not clear when we might begin to expect a detection. Recent quantitative predictions or constraints on the amplitude of GW emission associated with magnetar bursts are uncertain, and while the most recent are pessimistic (see e.g. [242-247]) these sources are still poorly understood. Furthermore, the closest is only about a kpc from Earth and precise sky locations and trigger times from electromagnetic (EM) bursts allow us to reduce the false-alarm rate and increase sensitivity relative to all-sky, all-time searches. GW might be emitted by damping of non-radial pulsational NS modes excited by a sudden localized energy release caused by untwisting of the global interior magnetic field and associated cracking of the solid NS crust [248], or global reconfiguration of the internal magnetic field and associated deformation of the NS hydrostatic equilibrium [242,245].

The $f$-mode is damped principally via $\mathrm{GW}$ emission and would ring down with a predicted damping time of $100-400 \mathrm{~ms}$ and with a frequency in the $1-3 \mathrm{kHz}$ range depending on the NS model [249]. If the magnetar outburst dynamics are confined to surface layer modes, torsional oscillations in the crust might emit GWs at frequencies of $\sim 10-2000 \mathrm{~Hz}$ [250]. It is possible that no neutron star mode will be excited at a sufficient level to emit detectable GWs; however, the lack of theoretical understanding implies the continued relevance of improving observational constraints on GW emission.

The physical mechanisms behind pulsar glitches are another possible route for excitation of GW-producing NS modes. Like the magnetar burst mechanism, the mechanism underlying pulsar glitches is also poorly understood. However, they might be caused by starquakes [251] or the transfer of angular momentum from a differentially rotating superfluid core to the solid star crust [252].

3.3.2. Continuous Sources of GWs: Spinning Deformed Neutron Stars. There are an estimated $10^{9}$ neutron stars in the galaxy (e.g., [253]), but only of order 3000 have been identified as radio/X-ray/ $\gamma$-ray sources. The detection of low-amplitude continuous GW emission in an all-sky search could mean the discovery of this large unseen neutron star population. Small nonaxisymmetric structures ("mountains") on neutron stars are the primary sources of continuous GWs that have been targeted by GW searches. Radio, X-ray, and $\gamma$-ray pulsars and spinning neutron stars in low-mass/high-mass X-ray binaries are primary potential sources. All-sky searches (which are presently computationally limited) for continuous signals could discover unknown, radio/X-ray $/ \gamma$-ray quiet neutron stars. Radio sources with precise timings can be followed up with long term integration that gains more than a magnitude in sensitivity.

The degree of nonaxisymmetry is typically quantified by the ellipticity $\epsilon=\left(I_{x x}-I_{y y}\right) / I_{z z}$ where $I_{i j}$ are components of the neutron star quadrupole moment. The fiducial gravitational strain from such a deformation will then be

$$
h_{0} \sim \frac{1}{D} \frac{G}{c^{4}}(2 \pi f)^{2} I_{z z} \epsilon,
$$

where $f=2 / P$ ( $P$ being the spin period of the neutron star) and $D$ is the distance to the detector. The signal will be sinusoidal with long-period modulations due to intrinsic properties of the source, binary orbital motion and motion of the detector relative to the source. Since emission is strongest for rapidly spinning sources, the detection of a low-frequency continuous wave source would be highly unexpected, but scientifically extremely rewarding.

Theory suggests an upper bound [254] on $\epsilon$ of

$$
\epsilon \lesssim 2 \times 10^{-5}\left(\frac{\sigma_{\text {break }}}{0.1}\right),
$$


where $\sigma_{\text {break }}$ is the breaking strain of the neutron star crust, which may be as large as $\sim 0.1$ [244]. However, regular radio pulsars are expected to have significantly smaller ellipticities of order $10^{-9}-10^{-8}$ [255], but young magnetars may exhibit significantly greater deformations (e.g., [253, 256]). Given these constraints on $\epsilon$ and Eq. (10) it is obvious that the expected GW strains will be minute even for nearby sources and can be found only by computationally intense long-term integrations. Constraining the ellipticity of known pulsars (e.g., Vela [257]) can constrain neutron star structure, crust physics, and pulsar models. Detection of continuous GWs from spinning neutron stars will provide interesting probes to the true nature of GWs. Having just one continuous wave source with moderately stable timing will place a very strong constraint on the speed of GWs, due to the large impact of Doppler corrections from detector movement.

For the purpose of this study, we assume that the continuous wave search pipeline PowerFlux [258] is being run on data from an ideal LIGO Voyager detector. In Fig 9 we provide estimates for the astrophysics range of the LIGO Voyager baseline design. The neutron star spindown is assumed to be solely due to GW emission, which is the most optimistic, astrophysically unrealistic case. For example, at the frequency of $1000 \mathrm{~Hz}$, assuming a modest ellipticity of few $\times 10^{-7}$ (which is well under the maximum limit in [244]) LIGO Voyager's reach will be almost $10 \mathrm{kpc}$. One should expect a gain of around another factor of 10 for a directed/targeted search.

The sensitivity of searches scales as $t^{1 / 2}$ for a coherent search down to $t^{1 / 4}$ for semi-coherent searches, where $t$ is the integration time. Thus, if one could make a change to the instrument that improved the sensitivity at frequencies above $1 \mathrm{kHz}$ by a factor of 3 and kept this running for 3 months it would be equivalent to running for more than 2 years for a coherent search (which is not practical for blind searches at all) and more than 10 years for a semi-coherent search.

There are two main methods of discovery of continuous wave sources: a search for unknown sources and a followup of pulsars discovered by radio/X-ray/ $\gamma$-ray surveys. Since the GW strain at fixed ellipticity increases with the square of the spin frequency, high frequency sources are easier to detect. On the other hand we know many more radio pulsars with periods of $1 \mathrm{~s}$ and higher than millisecond pulsars, but this sample is known to be highly biased.

For evaluating variations on the LIGO Voyager baseline design, we adopt three figures of merit:

(i) The integrated search volume of a PowerFlux-like blind search, where we consider the frequency space up to $1500 \mathrm{~Hz}$. This effectively assumes a flat prior on pulsar frequency. Keeping in mind that we do not know the distribution of pulsars with large ellipticity values this is not unreasonable. The volume is computed assuming the source has ellipticity $10^{-7}$ with emission frequency between 20 and $1500 \mathrm{~Hz}$.

(ii) An ellipticity bound placed by a targeted search for a high frequency pulsar with parameters similar to J1023+0038 (frequency $2 \times 592 \mathrm{~Hz}$, distance $900 \mathrm{pc}$ ). Smaller values of this figure of merit are better.

(iii) An ellipticity bound placed by a targeted search for a low frequency pulsar with parameters similar to Vela (frequency $2 \times 11.195 \mathrm{~Hz}$, distance $294 \mathrm{pc}$ ). Smaller values of this figure of merit are better.

The computation of these figures of merit uses a nominal constant to convert from design sensitivity to strain upper limits that could be obtained in actual search. The figure was derived by comparing initial LIGO design sensitivity to results of S5 run. This multiplicative factor is common to all numbers in Table 1 and cancels out for entries in Tables 2 and 3. 


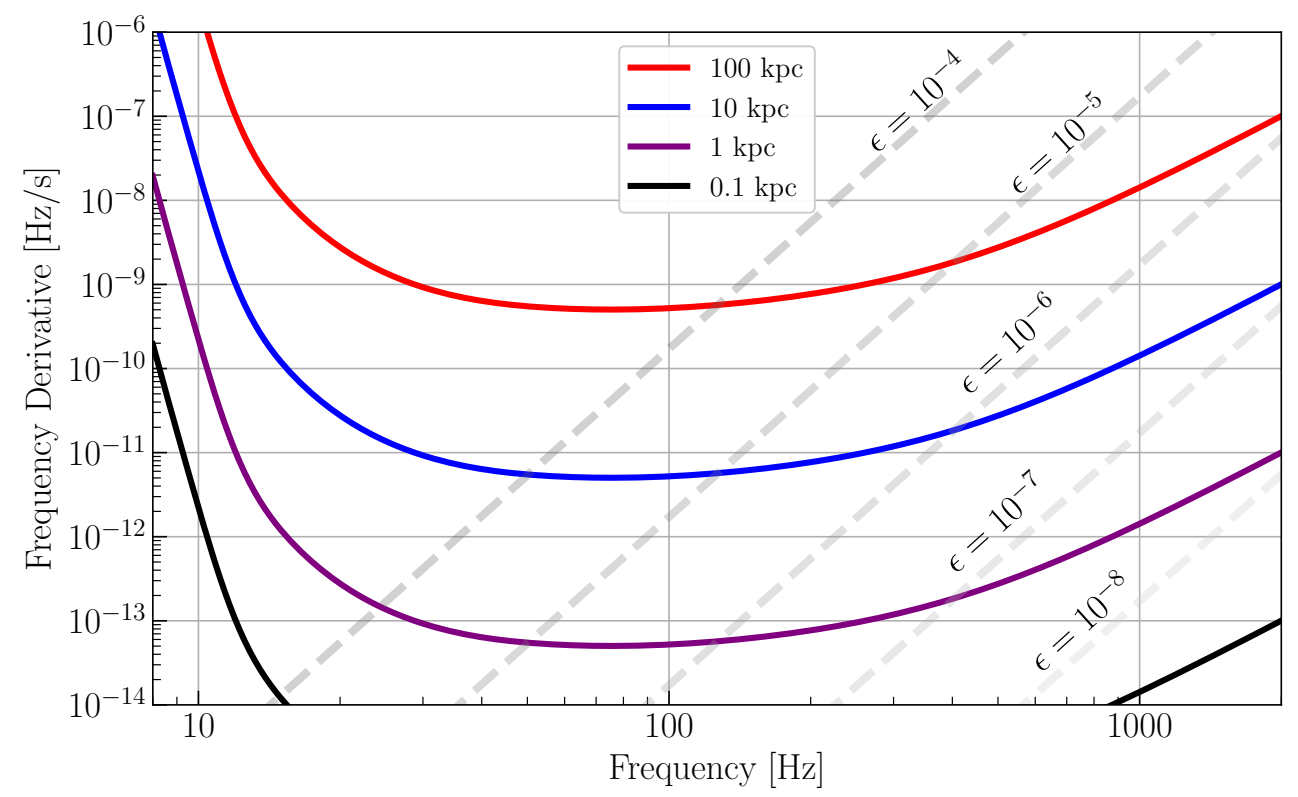

Figure 9: Range of the PowerFlux search for continuous gravitational waves from neutron stars spinning down solely due to gravitational radiation, assuming the baseline LIGO Voyager detector. This is a superposition of two contour plots. The solid lines are contours of the maximum distance at which a neutron star could be detected as a function of gravitational-wave frequency $f$ and its derivative $\dot{f}$. The dashed lines are contours of the corresponding ellipticity $\epsilon(f, \dot{f})$.

The results for these FOMs under variations of the baseline design are summarized in Tables 2 and 3. For first two FOMs we find the strongest improvement from noise reduction at high frequencies, such as obtained with increased squeezing or laser power. Table 1 shows that high-frequency targeted search can place very interesting bounds on pulsar signals - or make a detection. The third FOM benefits from any improvements to low frequency sensitivity, however it only approaches a region of interesting ellipticities.

3.3.3. Postmerger Oscillation Signal The most probable postmerger scenario following binary neutron star coalescence is the formation of a massive $\left(M>2 \mathrm{M}_{\odot}\right)$, differentially rotating neutron star [259-262]. The stability of this postmerger neutron star (PMNS) against gravitational collapse depends on its mass and on the details of the nuclear EOS. Less massive systems whose component masses add up to less than the maximum mass that can be supported by the EOS in combination with uniform rotation (the supramassive limit, e.g., [263, 264]), result in long-lived stable PMNSs. For more massive systems, strong differential rotation temporarily supports the remnant and it eventually undergoes gravitational collapse due to redistribution of angular momentum via viscous processes and radiation of GWs. The role of thermal pressure support is secondary, because at the densities involved, the temperature-insensitive pressure due to the nuclear force dominates the EOS [264]. Sufficiently high-mass systems that cannot be supported even by extreme differential rotation will result in prompt collapse to a black hole $(\mathrm{BH})$ upon merger or very shortly after merger, emitting a high-frequency ring-down gravitational wave (GW) signal at $\sim 6-7 \mathrm{kHz}$ (e.g., [259]).

Transient non-axisymmetric deformations in the surviving postmerger remnant lead to a 


\begin{tabular}{llllll}
\hline EoS & $\begin{array}{l}M_{\max } \\
{\left[\mathrm{M}_{\odot}\right]}\end{array}$ & $\begin{array}{l}R_{\max } \\
{[\mathrm{km}]}\end{array}$ & $\begin{array}{l}R_{1.35} \\
{[\mathrm{~km}]}\end{array}$ & $\rho_{\mathrm{c}} / \rho_{0}$ & $\begin{array}{c}f_{\text {peak }} \\
{[\mathrm{Hz}]}\end{array}$ \\
\hline APR [266] (approx) & 2.19 & 9.90 & 11.33 & 10.4 & 3405 \\
DD2 [267, 268] (full) & 2.42 & 11.90 & 13.21 & 7.2 & 2589 \\
Shen [269] (full) & 2.22 & 13.12 & 14.56 & 6.7 & 2263 \\
NL3 [268, 270] (full) & 2.79 & 13.43 & 14.75 & 5.6 & 2157 \\
\hline
\end{tabular}

Table 5: The nuclear EOS employed in this study. References are provided in the first column. EOS indicated by "approx" refer to models which rely on an approximate treatment of thermal effects, whereas "full" marks EOS which provide the full temperature dependence. $M_{\max }, R_{\max }$, and $\rho_{\mathrm{c}}$ are the gravitational mass, circumferential radius, and central energy density of the maximum-mass Tolman-OppenheimerVolkoff configurations. We list $\rho_{\mathrm{c}}$ in units of the nuclear saturation density $\rho_{0}=2.7 \times 10^{14} \mathrm{~g} \mathrm{~cm}^{-3} \cdot R_{1.35}$ is the circumferential radius of a $1.35 M_{\odot}$ NS. The final column $f_{\text {peak }}$ gives the dominant postmerger oscillation frequency.

short duration $(\sim 10-100 \mathrm{~ms})$ burst of GWs with rich high frequency content, dominated by emission from $f$-mode oscillations at $\sim 2-4 \mathrm{kHz}$ and generally lower-frequency subdominant peaks from nonlinear couplings between certain oscillation modes [265]. The general morphology of the GW signal thus emitted resembles an amplitude-modulated damped sinusoid, the phase and amplitude evolution of which are not yet well modeled by numerical simulations of neutron star coalescence and postmerger evolution. However, the spectral properties of this signal carry a particularly distinct signature of the EOS.

A number of studies [262, 271, 272] have identified and confirmed a correlation between the dominant postmerger oscillation frequency (i.e., half the peak GW emission frequency) and the radius of a fiducial cold, non-rotating neutron star. For example, in [262], the authors perform a Fisher matrix analysis and find that it may be possible to use aLIGO observations of postmerger signals to measure the dominant postmerger oscillation frequency to an accuracy of $\sim 40 \mathrm{~Hz}$ and thus determine the radius of a fiducial $1.6 \mathrm{M}_{\odot} \mathrm{NS}$ to an accuracy of 100-200 m with an expected detection horizon of approximately $15 \mathrm{Mpc} *$, corresponding to a detection rate of just under 1 per century, assuming the "realistic" rate in [42]. Furthermore, a systematic Monte-Carlo analysis using a variety of postmerger waveforms corresponding to different component masses and EOS and data from the initial-LIGO/Virgo instruments, recolored to the nominal advanced detector design sensitivities was presented in [273]. There, the results of [262] are essentially confirmed, albeit at a reduced expected detection range of $\sim 5 \mathrm{Mpc}$, where this is now the angle-averaged range since a network of detectors was used in that analysis and so the notion of horizon distance is not well-defined. This corresponds to an expected detection rate of $\sim 0.5$ events per century. The reduction in sensitivity arises since a generic burst search was used in the absence of accurate templates. Fisher analysis, by contrast, assumes that an optimal filtering strategy is feasible.

Here, we estimate the detectability of the postmerger signal from a surviving PMNS for four different EOS using three figures of merit: the angle-averaged signal-to-noise ratio, described in A; the horizon distance, the distance at which an optimally-oriented face-on source yields an optimal matched-filter SNR of some fiducial value and the expected annual detection rate based on the "realistic" rates from [42]†. Our interest here is in the detectability of the merger/postmerger signal arising from surviving PMNS. We therefore choose to window the waveforms prior to computing their optimal and angle-averaged SNR such that the time-

*Assuming an optimal SNR detection threshold of 5, justified below.

$\dagger$ As with the estimates for core-collapse supernovae, these FOMs assume that the waveform is sufficiently wellmodeled to permit a matched filtering detection strategy. 


\begin{tabular}{|c|c|c|c|c|c|c|c|c|c|c|c|c|c|}
\hline \multirow[b]{2}{*}{ EoS } & \multicolumn{3}{|c|}{ aLIGO } & \multicolumn{3}{|c|}{ Blue LIGO Voyager Baseline } & \multicolumn{3}{|c|}{ Red LIGO Voyager Baseline } & \multicolumn{3}{|c|}{ Green LIGO Voyager Baseline } & \multirow{2}{*}{$\begin{array}{c}\text { Frequency Recovery } \\
\tilde{\delta f} \pm \mathrm{IQR} \\
{[\mathrm{Hz}]} \\
\end{array}$} \\
\hline & $\begin{array}{c}\langle\rho\rangle \\
@ 10 \mathrm{Mpc}\end{array}$ & $\begin{array}{c}D_{\mathrm{H}} \\
{[\mathrm{Mpc}]}\end{array}$ & $\begin{array}{c}\dot{N} \\
{\left[\mathrm{yr}^{-1}\right]}\end{array}$ & $\begin{array}{c}\langle\rho\rangle \\
@ 10 \mathrm{Mpc}\end{array}$ & $\begin{array}{c}D_{\mathrm{H}} \\
{[\mathrm{Mpc}]}\end{array}$ & $\begin{array}{c}\dot{\mathcal{N}} \\
{\left[\mathrm{yr}^{-1}\right]}\end{array}$ & $\begin{array}{c}\langle\rho\rangle \\
\text { @ } 10 \mathrm{Mpc}\end{array}$ & $\begin{array}{c}D_{\mathrm{H}} \\
{[\mathrm{Mpc}]}\end{array}$ & $\begin{array}{c}\dot{\mathcal{N}} \\
{\left[\mathrm{yr}^{-1}\right]}\end{array}$ & $\begin{array}{c}\langle\rho\rangle \\
@ 10 \mathrm{Mpc}\end{array}$ & $\begin{array}{c}D_{\mathrm{H}} \\
{[\mathrm{Mpc}]}\end{array}$ & $\underset{\substack{\dot{N} \\
\left[\mathrm{yr}^{-1}\right]}}{ }$ & \\
\hline $\mathrm{AP}$ & 1. & 8.3 & & 5 & 39.6 & 0.09 & 4.7 & 33 & 0.05 & 9.1 & & & \\
\hline DD2 & 2. & 14.1 & & 9.1 & 53 & 0.2 & 8.0 & 52 & 0. & 14 & 94 & 0. & \\
\hline Shen & 2. & 13.8 & & 9.37 & 70. & 0.43 & 8.3 & 54.08 & 0.2 & 14.73 & 94.81 & 0.7 & $8 \pm 22$ \\
\hline NL3 & 3.13 & 15.7 & $7.7 \times 10^{-3}$ & 11.15 & 73.84 & 0.49 & 9.91 & 67.04 & 0.37 & 17.26 & 115.03 & 0.74 & $5 \pm 10$ \\
\hline
\end{tabular}

Table 6: Detectability of the post-merger oscillation in binary neutron star mergers. Angle-averaged SNR $\langle\rho\rangle$, horizon distances $D_{\mathrm{H}}$, and expected annual detection rate $\dot{\mathcal{N}}$ (at SNR $=5$ ). Local merger rates are based on "realistic" rates [42], but are uncertain within three orders of magnitude. Note that the true SNR recovered by a burst search will be factors of a few below the values given here (which are based on matched filtering). We also estimate the accuracy in the determination of the dominant postmerger oscillation frequency, based on a Monte-Carlo analysis (see text for details).

domain amplitude of the waveform is zero at times prior to the merger (taken as the point of maximum amplitude). This helps to ensure that there is essentially no contribution to the SNR (and hence, our detectability estimates) from the pre-merger inspiral signal. Finally, the SNR is computed for frequencies of $1 \mathrm{kHz}$ and above.

Since the postmerger signal is likely to only be observable for relatively nearby, rare events, it is reasonable to assume that its time is known to extremely high accuracy from the time of coalescence measured from the much higher SNR inspiral precursor. We therefore adopt a relatively low nominal SNR threshold of 5 in computing the horizon distance and detection rate. The EOS used for these estimates are a subset of those used in the more extensive study of [273] and range from the rather soft APR (high frequency GW signal) to the somewhat softer NL3 (lower frequency GW signal). Table 6 reports the FOMs described above for each waveform, for both the aLIGO noise curve and that of the red, blue, and green LIGO Voyager baseline designs.

While the mere detection of the postmerger signal will itself have significant consequences for our understanding of the neutron star EOS by excluding those EOS unable to support a long-lived postmerger object, one of the most useful, and simple, measurements that will be possible is the identification of the dominant postmerger oscillation frequency. It is, therefore, informative to also estimate the accuracy with which the postmerger frequency may be determined. To that end, we have performed a Monte-Carlo study wherein a population of postmerger waveforms with a uniform distribution in SNR have been injected into Gaussian noise with the noise spectral density of the blue LIGO Voyager baseline design. The signals are recovered using a Bayesian nested sampling algorithm with a simple sine-Gaussian template and the peak frequency of the postmerger signal is recovered from the maximumlikelihood estimate (ML) of the sine-Gaussian template. This is a reasonable approximation to a realistic burst search; the sine-Gaussian yields a 70\% match, or better, with the postmerger waveforms and allows for accurate recovery of the peak frequency. We quantify the accuracy of the frequency estimation via the error $\delta f=f_{\text {True }}-f_{\mathrm{ML}}$. Table 6 reports the median value and the interquartile range $*$ of the frequency error for each waveform, for all injections recovered with a sine-Gaussian SNR $>6$. This higher threshold is chosen to reflect the fact that this burst-like analysis will recover a factor of order unity less of the SNR that an optimal matchedfilter strategy would. Since this frequency estimate is essentially only SNR-dependent (i.e., the differences in the shapes of the aLIGO and LIGO Voyager baseline design noise curves at such high frequencies do not affect this analysis), the frequency error is common to both noise curves.

In conclusion, we find that the blue LIGO Voyager baseline design will provide a realistic

$*$ the difference between the $25^{\text {th }}$ and $75^{\text {th }}$ percentiles, representing a robust measure of the spread in the frequency error. 


\begin{tabular}{lcccccccc}
\hline Waveform & NN & Sei & SUS & SPOT/CTN & SQZ & POW & FCL & MASS \\
\hline APR & 0 & 0 & $4.50 \times 10^{-7}$ & $4.89 \times 10^{-3}$ & 0.81 & 0.45 & $-2.73 \times 10^{-5}$ & $-2.79 \times 10^{-5}$ \\
DD2 & 0 & 0 & $1.62 \times 10^{-7}$ & $7.89 \times 10^{-3}$ & 0.81 & 0.45 & $-4.14 \times 10^{-5}$ & $-4.26 \times 10^{-5}$ \\
Shen & 0 & 0 & $1.46 \times 10^{-7}$ & 0.01 & 0.81 & 0.45 & $-5.18 \times 10^{-5}$ & $-5.31 \times 10^{-5}$ \\
NL3 & 0 & 0 & $1.83 \times 10^{-8}$ & 0.01 & 0.81 & 0.45 & $-5.76 \times 10^{-5}$ & $-5.93 \times 10^{-5}$ \\
\hline \hline
\end{tabular}

Table 7: Jacobian of science goals as a function of interferometer upgrade technology. The baseline FOM is the ideal optimally-oriented single-detector matched filtering SNR for various postmerger waveforms (cf. Section 3.3.3). The true SNR recovered by a burst search will be factors of order unity below the values given for aLIGO and the baseline LIGO Voyager design.

chance of the detection, and characterization, of the GW signal associated with postmerger oscillations following binary NS coalescence. A search for these signals with data from LIGO Voyager would still require some level of optimism and a relatively nearby (i.e., $10-50 \mathrm{Mpc}$ ) event, but, given the uncertainties in the expected rate of binary NS coalescence in the local Universe [42], the uncertainties in the numerical modeling of the postmerger signal and the science possible with the detection and accurate measurement of the dominant postmerger oscillation frequency $(\delta f \sim 10 \mathrm{~Hz}$ ), this constitutes an important high-frequency source for the next generation GW observatories.

\subsection{Stochastic Background and Unanticipated Discoveries}

The LIGO Voyager detector could be used to look for a few different types of stochastic background radiation: spatially resolved regions of space with quasi-periodic or quasicontinuous radiation of a random nature, an unresolved foreground of astrophysical sources, and a background of cosmological origin. This has already been previously explored within the context of the Einstein Telescope Design study [274].

For the purposes of optimizing the design of this detector, we do not include stochastic sources in the cost function. The expected cosmological background from inflation is either too weak to detect [275] or is non-existent [276, 277]. Other cosmological stochastic background sources, such as early-Universe strongly first-order phase transitions, also suffer from uncertain amplitudes and peak frequencies [278]. Estimates of the astrophysical foregrounds [279] have significant error bars at present, and work is still ongoing to quantify the scientific benefits of observing these foregrounds [280]. Therefore, stochastic backgrounds do not influence the detector design in any quantitative way at present. Qualitatively, we remain excited about the prospect of making serendipitous discoveries of heretofore unforeseen stochastic sources.

Whenever a new vista onto the cosmos has been exposed in the past, it has revolutionized our understanding of the Universe and its denizens. We anticipate a similarly dramatic upheaval as the gravitational wave Universe reveals itself. A detector with broadband sensitivity is best suited for exploring the full range of serendipitous discoveries. 


\section{Conclusions}

We have shown that a number of significant quantitative improvements can be achieved relative to a wide array of known astrophysical targets by upgrading the LIGO interferometers within the existing facilities. Precision tests of extreme spacetime curvatures can be made with these improved instruments, perhaps even shedding light on what really happens at the black hole horizons. In order to aid with making design tradeoffs for the LIGO Voyager detector, we have numerically computed derivatives for these targets, indicating how much scientific value there is in incremental improvements in the interferometers. It is clear from the Jacobian tables that there are significant astrophysical gains to be made for modest investments in the reduction of technical noise in the audio band $(40-8000 \mathrm{~Hz})$. To make improvements for the low frequency $(10-40 \mathrm{~Hz})$ science targets (e.g. GW memory or mergers of higher mass black holes) would require order-of-magnitude improvements in the seismic isolation, suspension thermal noise, and Newtonian gravity noise.

This work should serve as a guide in making these detector design choices as well a starting point for more exhaustive evaluation of other science targets.

\section{Acknowledgements}

RXA, PA and IM performed part of this work at the Aspen Center for Physics, which is supported by National Science Foundation grant PHY-1066293. JAC acknowledges support under NSF PHYS-1505824 and PHYS-1505524. JSR acknowledges support from NSF PHYS-1307545 and the Research Corporation for Science Advancement. RXA, PA and YC acknowledge support from the Indo-US Centre for the Exploration of Extreme Gravity funded by the Indo-US Science and Technology Forum (IUSSTF/JC-029/2016). In addition, P. A.'s research was supported by a Ramanujan Fellowship from the Science and Engineering Research Board (SERB), India, and by the Max Planck Society through a Max Planck Partner Group at ICTS. 


\section{A. Estimating Signal-To-Noise Ratios}

The gravitational-wave signal $h(t)$ in an interferometric detector is given by

$$
h(t)=F_{+}(\theta, \varphi, \Psi) h_{+}(t, D, \iota, \beta)+F_{\times}(\theta, \varphi, \Psi) h_{\times}(t, D, \iota, \beta) .
$$

$F_{+}$and $F_{\times}$are the interferometer beam pattern functions [281]. $h_{+}$and $h_{\times}$are the two independent polarizations of the gravitational-wave strain amplitude. The source is located at distance $D$ and position $(\theta, \varphi)$ (spherical polar angles) on the sky. In the reference frame of the source, Earth is located at a position $(\iota, \beta)$ (spherical polar angles). $\Psi$ is the angle of the waves' polarization axis on the plane of the sky relative to the detector's orientation (see Fig. 9.2 of [281]).

The optimal Wiener matched-filter signal-to-noise ratio $(\mathrm{SNR}, \rho)$ is

$$
\rho^{2}=4 \int_{0}^{\infty} \frac{|\tilde{h}(f)|^{2}}{S(f)} d f
$$

where

$$
\tilde{h}(f)=\int_{-\infty}^{\infty} h(t) \exp (2 \pi i f t) d f,
$$

and $S(f)$ is the one-sided power spectral density of noise in the detector.

Since sky location, orientation of the source, and polarization angle are unknown, it is most meaningful to compute and state SNRs that are averages over these angles. In this, we follow Flanagan \& Hughes [282] and use their equation (2.30), which gives the angle-averaged SNR in terms of the spectral energy density of the waves

$$
\left\langle\rho^{2}\right\rangle=\frac{2}{5 \pi^{2} D^{2}} \frac{G}{c^{3}} \int_{0}^{\infty} d \ln f \frac{1}{f S(f)} \frac{d E_{\mathrm{GW}}}{d f} .
$$

When comparing GW spectra with LIGO 3 detector design sensitivity curves (the one-sided amplitude spectral density $\sqrt{S(f)}$ ), we plot

$$
\bar{h}(f)=\sqrt{\frac{2}{5 \pi^{2} D} \frac{G}{c^{3}} \frac{1}{f} \frac{d E_{\mathrm{GW}}}{d f}},
$$

which has dimension of (frequency) $)^{-1 / 2}$ and thus can be compared directly with $\sqrt{S(f)}$ : the ratio of $\bar{h}$ and $\sqrt{S(f)}$ is dimensionless. 


\section{B. Interferometer Parameters}

\begin{tabular}{lccc}
\hline Parameter & Advanced LIGO & Red & Blue \\
\hline Laser wavelength & $1064 \mathrm{~nm}$ & $1064 \mathrm{~nm}$ & $2000 \mathrm{~nm}$ \\
Laser power & $125 \mathrm{~W}$ & $125 \mathrm{~W}$ & $151.5919 \mathrm{~W}$ \\
Mirror substrate & fused silica & fused silica & silicon \\
Mirror radius & $17 \mathrm{~cm}$ & $27.5 \mathrm{~cm}$ & $22.5 \mathrm{~cm}$ \\
Mirror thickness & $20 \mathrm{~cm}$ & $30.6 \mathrm{~cm}$ & $55 \mathrm{~cm}$ \\
Beam radius on ITM/ETM & $5.5 / 6.2 \mathrm{~cm}$ & $16.9 / 19.9 \mathrm{~cm} *$ & $5.9 / 8.4 \mathrm{~cm}$ \\
Mass per stage & $22.1 / 21.8 / 39.6 / 39.6 \mathrm{~kg}$ & $44 / 66 / 120 / 160 \mathrm{~kg}$ & $59.1534 / 60.8466 / 200 / 200 \mathrm{~kg}$ \\
Final stage temperature & $300 \mathrm{~K}$ & $290 \mathrm{~K}$ & $123 \mathrm{~K}$ \\
Final stage construction & fused silica fiber & fused silica fiber $\dagger$ & silicon ribbon \\
Final stage length & $0.602 \mathrm{~m}$ & $1.2 \mathrm{~m}$ & $0.69685 \mathrm{~m}$ \\
Newtonian noise suppression & N/A & 5 & 10 \\
Squeeze factor & N/A & $10 \mathrm{~dB} \neq$ & $10 \mathrm{~dB}$ \\
Squeeze injection loss & N/A & 0.05 & 0.05 \\
Squeeze filter cavity length & N/A & $300 \mathrm{~m}$ & $300 \mathrm{~m}$ \\
Squeeze filter cavity loss & N/A & $40 \mathrm{ppm}-\mathrm{rt}$ & $10 \mathrm{ppm}-\mathrm{rt}$ \\
\hline
\end{tabular}

Table 8: Parameters varied by the Red and Blue designs, relative to Advanced LIGO.

\section{References}

[1] Abbott B P, Abbott R, Abbott T D, Abernathy M R, Acernese F et al. (LIGO Scientific Collaboration and Virgo Collaboration) 2016 Phys. Rev. Lett. 116(6) 061102 URL http: //link.aps.org/doi/10.1103/PhysRevLett. 116.061102

[2] The LIGO Scientific Collaboration, the Virgo Collaboration, Abbott B P, Abbott R, Abbott T D, Abraham S, Acernese F, Ackley K, Adams C, Adhikari R X and et al 2018 arXiv e-prints (Preprint 1811.12940)

[3] Abbott B P, Abbott R, Abbott T D, Acernese F, Ackley K, Adams C, Adams T, Addesso P, Adhikari R X, Adya V B and et al 2017 Physical Review Letters 119161101 (Preprint 1710.05832)

[4] Adhikari R, Gustafson E K, Hild S, Ballmer S and Arai K 2012 Report of the Third Generation LIGO Strawman Design Meeting Tech. rep. LIGO Scientific Collaboration URL https : //dcc . ligo . org/cgi-bin/private/DocDB/ ShowDocument?docid $=85610$

[5] Collaboration L S 2016 LSC Instrument Science White Paper Tech. rep. LIGO Scientific Collaboration URL https: //dcc . ligo . org/LIGO-T1600119/public

[6] Adhikari R 2019 A cryogenic interferometer for gravitational-wave detection in prep.

[7] Punturo M, Abernathy M, Acernese F, Allen B, Andersson N and andothers K A 2010 Classical and Quantum Gravity 27084007

*The Red design projects a factor of 3.2 improvement in all coating and substrate noises. A factor of 1.6 is to be achieved through increasing the beam size, and a further factor of 2 through coating technology improvements. For the noise budget, this has been modeled as an increase in the beam size by the full factor of 3.2.

$\dagger$ The noise of the optimized suspension fiber in the Red design was roughly fitted in GWINC, using the specific heat of fused silica as the fit parameter.

$\ddagger$ Squeezer parameters have been altered to provide an equal ground for comparison between the two designs. T1200005 originally assumed $20 \mathrm{~dB}$ of squeezing. 
[8] Abbott B P, Abbott R, Abbott T, Abernathy M, Ackley K, Adams C, Addesso P, Adhikari R, Adya V, Affeldt C et al. 2017 Classical and Quantum Gravity 34044001

[9] Cumming A, Heptonstall A, Kumar R, Cunningham W, Torrie C, Barton M, Strain K A, Hough J and Rowan S 2009 Classical and Quantum Gravity 26215012 URL http: //stacks . iop. org/0264-9381/26/i=21/a=215012

[10] Cumming A V, Bell A S, Barsotti L, Barton M A, Cagnoli G, Cook D, Cunningham L, Evans M, Hammond G D, Harry G M, Heptonstall A, Hough J, Jones R, Kumar R, Mittleman R, Robertson N A, Rowan S, Shapiro B, Strain K A, Tokmakov K, Torrie C and van Veggel A A 2012 Classical and Quantum Gravity 29035003 URL http: //stacks . iop .org/0264-9381/29/i=3/a=035003

[11] Abbott B P, Abbott R, Abbott T D, Abernathy M R, Acernese F et al. (LIGO Scientific Collaboration and Virgo Collaboration) 2016 Phys. Rev. Lett. 116(13) 131103 URL http: //link.aps .org/doi/10.1103/PhysRevLett. 116.131103

[12] Miller J, Barsotti L, Vitale S, Fritschel P, Evans M and Sigg D 2015 Phys. Rev. D 91(6) 062005 URL https: //link . aps .org/doi/10.1103/PhysRevD . 91.062005

[13] Harms J 2015 ArXiv e-prints (Preprint 1507 . 05850)

[14] Matichard F, Lantz B, Mittleman R, Mason K, Kissel J, Abbott B et al. 2015 Classical and Quantum Gravity 32185003 URL http://stacks. iop.org/0264-9381/32/ $i=18 / \mathrm{a}=185003$

[15] Cumming A V, Cunningham L, Hammond G D, Haughian K, Hough J, Kroker S, Martin I W, Nawrodt R, Rowan S, Schwarz C and van Veggel A A 2014 Classical and Quantum Gravity 31025017 URL http: //stacks . iop. org/0264-9381/31/i=2/ $\mathrm{a}=025017$

[16] Reid S and Martin I W 2016 Coatings 661

[17] Miao H, Yang H, Adhikari R X and Chen Y 2014 Classical and Quantum Gravity 31 165010 URL http: //stacks . iop . org/0264-9381/31/i=16/a=165010

[18] Evans M, Barsotti L, Kwee P, Harms J and Miao H 2013 Phys. Rev. D 88(2) 022002 URL https: //link . aps . org/doi/10.1103/PhysRevD . 88.022002

[19] Mandel I and O'Shaughnessy R 2010 Classical and Quantum Gravity 27114007 (Preprint 0912 . 1074)

[20] Abbott B P, Abbott R, Abbott T D, Abernathy M R, Acernese F, Ackley K, Adams C, Adams T, Addesso P, Adhikari R X and et al 2016 Astrophys. J. Lett. 818 L22 (Preprint 1602 . \$3846)

[21] Mandel I and Farmer A 2018 ArXiv e-prints (Preprint 1806.05820)

[22] Mapelli M 2018 arXiv e-prints (Preprint 1809.09130)

[23] Kalogera V, Belczynski K, Kim C, O’Shaughnessy R and Willems B 2007 Physics Reports 442 75-108 (Preprint astro-ph/0612144)

[24] Postnov K A and Yungelson L R 2014 Living Reviews in Relativity 173 (Preprint $1403.4754)$

[25] Tauris T M, Kramer M, Freire P C C, Wex N, Janka H T, Langer N, Podsiadlowski P, Bozzo E, Chaty S, Kruckow M U, van den Heuvel E P J, Antoniadis J, Breton R P and Champion D J 2017 Astrophys. J. 846170 (Preprint 1706. 09438)

[26] Belczynski K, Kalogera V, Rasio F A, Taam R E, Zezas A, Bulik T, Maccarone T J and Ivanova N 2008 Astrophys. J. Suppl. Ser. 174 223-260 
[27] Pfahl E, Podsiadlowski P and Rappaport S 2005 Astrophys. J. 628 343-352 (Preprint astro-ph/0502122)

[28] Voss R and Tauris T M 2003 Mon. Not. R. Astron. Soc. 342 1169-1184 (Preprint astro-ph/0303227)

[29] Dominik M, Berti E, O’Shaughnessy R, Mandel I, Belczynski K, Fryer C, Holz D E, Bulik T and Pannarale F 2015 Astrophys. J. 806263 (Preprint 1405.7016)

[30] Vigna-Gómez A, Neijssel C J, Stevenson S, Barrett J W, Belczynski K, Justham S, de Mink S E, Müller B, Podsiadlowski P, Renzo M, Szécsi D and Mandel I 2018 Mon. Not. R. Astron. Soc. 481 4009-4029 (Preprint 1805.07974)

[31] Belczynski K, Holz D E, Bulik T and O’Shaughnessy R 2016 Nature 534 512-515 (Preprint 1602.04531)

[32] Eldridge J J and Stanway E R 2016 ArXiv e-prints (Preprint 1602 .03790)

[33] Lipunov V M, Kornilov V, Gorbovskoy E, Tiurina N, Balanutsa P and Kuznetsov A 2016 ArXiv e-prints (Preprint 1605.01604)

[34] Stevenson S, Vigna-Gómez A, Mandel I, Barrett J W, Neijssel C J, Perkins D and de Mink S E 2017 Nature Communications 814906 (Preprint 1704.01352)

[35] Mandel I and de Mink S E 2016 Mon. Not. R. Astron. Soc. 458 2634-2647 (Preprint 1601.00007)

[36] Marchant P, Langer N, Podsiadlowski P, Tauris T M and Moriya T J 2016 Astron. Astrophys. 588 A50 (Preprint 1601.03718)

[37] Bird S, Cholis I, Muñoz J B, Ali-Haïmoud Y, Kamionkowski M, Kovetz E D, Raccanelli A and Riess A G 2016 Physical Review Letters 116201301 (Preprint 1603.00464)

[38] Sasaki M, Suyama T, Tanaka T and Yokoyama S 2016 Phys. Rev. Lett. 117061101 (Preprint 1603.08338)

[39] Rodriguez C L, Haster C J, Chatterjee S, Kalogera V and Rasio F A 2016 Astrophys. J. Lett. 824 L8 (Preprint 1604.04254)

[40] Stone N C, Metzger B D and Haiman Z 2017 Mon. Not. R. Astron. Soc. $464946-954$ (Preprint 1602.04226)

[41] Bartos I, Kocsis B, Haiman Z and Márka S 2016 ArXiv e-prints (Preprint 1602 . 03831)

[42] Abadie J et al. (LIGO Scientific Collaboration and Virgo Collaboration) 2010 Classical and Quantum Gravity 27 173001-+ (Preprint 1003.2480)

[43] Abbott B P, Abbott R, Abbott T D, Acernese F, Ackley K, Adams C, Adams T, Addesso P, Adhikari R X, Adya V B and et al 2017 Astrophys. J. Lett. 848 L12 (Preprint 1710.05833)

[44] Abbott B P, Abbott R, Abbott T D, Abernathy M R, Acernese F, Ackley K, Adams C, Adams T, Addesso P, Adhikari R X and et al 2016 Physical Review Letters 116241102 (Preprint 1602.03840)

[45] Veitch J, Raymond V, Farr B, Farr W, Graff P, Vitale S, Aylott B, Blackburn K, Christensen N, Coughlin M, Del Pozzo W, Feroz F, Gair J, Haster C J, Kalogera V, Littenberg T, Mandel I, O'Shaughnessy R, Pitkin M, Rodriguez C, Röver C, Sidery T, Smith R, Van Der Sluys M, Vecchio A, Vousden W and Wade L 2015 Phys. Rev. D. 91 042003 (Preprint 1409.7215)

[46] Vitale S and Evans M 2017 Phys. Rev. D. 95064052 (Preprint 1610.06917) 
[47] Rodriguez C L, Chatterjee S and Rasio F A 2016 Phys. Rev. D. 93084029 (Preprint 1602.02444)

[48] Stevenson S, Ohme F and Fairhurst S 2015 Astrophys. J. 81058 (Preprint 1504. Q7802)

[49] Stevenson S, Berry C P L and Mandel I 2017 ArXiv e-prints (Preprint 1703. 06873)

[50] Barrett J W, Gaebel S M, Neijssel C J, Vigna-Gómez A, Stevenson S, Berry C P L, Farr W M and Mandel I 2018 Mon. Not. R. Astron. Soc. 477 4685-4695 (Preprint 1711.06287)

[51] Mandel I, Haster C J, Dominik M and Belczynski K 2015 Mon. Not. R. Astron. Soc. 450 L85-L89 (Preprint 1503.03172)

[52] Vitale S, Lynch R, Sturani R and Graff P 2017 Classical and Quantum Gravity 34 03LT01 (Preprint 1503.04307)

[53] Mandel I, Farr W M, Colonna A, Stevenson S, Tiňo P and Veitch J 2017 Mon. Not. R. Astron. Soc. 465 3254-3260 (Preprint 1608.08223)

[54] Farr W M, Stevenson S, Miller M C, Mandel I, Farr B and Vecchio A 2017 ArXiv e-prints (Preprint 1706.01385)

[55] Read J S, Markakis C, Shibata M, Uryū K, Creighton J D E and Friedman J L 2009 Phys. Rev. D. 79 124033-+ (Preprint arXiv: 0901.3258)

[56] Abbott B P, Abbott R, Abbott T D, Acernese F, Ackley K, Adams C, Adams T, Addesso P, Adhikari R X, Adya V B and et al 2017 Astrophys. J. Lett. 848 L13 (Preprint 1710.05834)

[57] Cannon K, Cariou R, Chapman A, Crispin-Ortuzar M, Fotopoulos N, Frei M, Hanna C, Kara E, Keppel D, Liao L, Privitera S, Searle A, Singer L and Weinstein A 2012 Astrophys. J. 748136 (Preprint 1107.2665)

[58] Miller M C and Colbert E J M 2004 International Journal of Modern Physics D 13 1-64 (Preprint arXiv: astro-ph/0308402)

[59] Farrell S A, Webb N A, Barret D, Godet O and Rodrigues J M 2009 Nature 460 73-75

[60] Abadie J et al. (LIGO Scientific Collaboration and Virgo Collaboration) 2011 Submitted to PRD (Preprint arXiv: 1102 .3781)

[61] Veitch J, Pürrer M and Mandel I 2015 Physical Review Letters 115141101 (Preprint 1503.05953)

[62] Gair J R, Mandel I, Miller M C and Volonteri M 2011 General Relativity and Gravitation 43 485-518 (Preprint 0907.5450)

[63] Mandel I, Brown D A, Gair J R and Miller M C 2008 Astrophys. J. 681 1431-1447 (Preprint 0705.0285)

[64] MacLeod M, Trenti M and Ramirez-Ruiz E 2016 Astrophys. J. 81970 (Preprint $1508.07000)$

[65] Haster C J, Antonini F, Kalogera V and Mandel I 2016 Astrophys. J. 832192 (Preprint 1606.07097)

[66] Fregeau J M, Larson S L, Miller M C, O'Shaughnessy R and Rasio F A 2006 Astrophysical Journal Letters 646 L135-L138 (Preprint astro-ph/0605732)

[67] Amaro-Seoane P and Santamaría L 2010 Astrophys. J. 722 1197-1206 (Preprint 0910.0254)

[68] Madau P and Rees M J 2001 Astrophys. J. Lett. 551 L27-L30 
[69] Sesana A, Gair J, Mandel I and Vecchio A 2009 Astrophys. J. Lett. 698 L129-L132 (Preprint 0903.4177)

[70] Gair J R, Mandel I, Sesana A and Vecchio A 2009 Classical and Quantum Gravity 26 204009 (Preprint 0907 . 3292)

[71] Will C 2001 Living Reviews in Relativity 44 (Preprint arXiv: gr-qc/0103036)

[72] Yunes N 2011 ArXiv e-prints (Preprint 1112 . 3694)

[73] Abbott B P, Abbott R, Abbott T D, Abernathy M R, Acernese F, Ackley K, Adams C, Adams T, Addesso P, Adhikari R X and et al 2016 Physical Review Letters 116221101 (Preprint 1602.03841)

[74] Will C M 2006 Living Reviews in Relativity 9 URL http://www. livingreviews. org/lrr-2006-3

[75] Will C M 1998 Phys. Rev. D 57 2061-2068

[76] Abbott B P et al. (LIGO Scientific and Virgo Collaborations) 2016 Phys. Rev. Lett. 116(22) 221101 URL https: //link. aps .org/doi/10.1103/PhysRevLett. 116. 221101

[77] Keppel D and Ajith P 2010 ArXiv:1004.0284 [gr-qc] (Preprint 1004 . 0284)

[78] Arun K G, Iyer B R, Qusailah M S S and Sathyaprakash B S 2006 Classical and Quantum Gravity 23 L37-L43 (Preprint arXiv: gr-qc/0604018)

[79] Mishra C K, Arun K G, Iyer B R and Sathyaprakash B S 2010 Phys. Rev. D. 82064010 (Preprint 1005.0304)

[80] Li T G F, Del Pozzo W, Vitale S, Van Den Broeck C, Agathos M, Veitch J, Grover K, Sidery T, Sturani R and Vecchio A 2011 ArXiv e-prints (Preprint 1110.0530)

[81] Blanchet L and Schafer G 1993 Classical and Quantum Gravity 10 2699-2721 URL https: //doi .org/10 . 1088\%2F0264-9381\%2F 10\%2F 12\%2FQ26

[82] Blanchet L and Le Tiec A 2017 Classical and Quantum Gravity 34164001

[83] Favata M 2009 Phys. Rev. D 80(2) 024002 URL http://link.aps.org/doi/10. 1103/PhysRevD.80.024002

[84] Favata M 2010 Classical and Quantum Gravity 27084036 (Preprint 1003. 3486)

[85] Pollney D and Reisswig C 2011 The Astrophysical Journal Letters 732 L13 URL http://stacks.iop.org/2041-8205/732/i=1/a=L13

[86] Talbot C, Thrane E, Lasky P D and Lin F 2018 Phys. Rev. D 98(6) 064031 URL https://link.aps.org/doi/10.1103/PhysRevD.98.064031

[87] Lasky P D, Thrane E, Levin Y, Blackman J and Chen Y 2016 Physical Review Letters 117061102 (Preprint 1605.01415)

[88] Yang H and Martynov D 2018 Phys. Rev. Lett. 121(7) 071102 URL https://link. aps.org/doi/10.1103/PhysRevLett.121.071102

[89] Yu H, Martynov D, Vitale S, Evans M, Shoemaker D, Barr B, Hammond G, Hild S, Hough J, Huttner S, Rowan S, Sorazu B, Carbone L, Freise A, Mow-Lowry C, Dooley K L, Fulda P, Grote H and Sigg D 2018 Physical Review Letters 120141102 (Preprint 1712.05417)

[90] Ryan F D 1995 Phys. Rev. D52 5707-5718

[91] Meidam J, Agathos M, Van Den Broeck C, Veitch J and Sathyaprakash B S 2014 Phys. Rev. D. 90064009 (Preprint 1406.3201) 
[92] Brown D A, Brink J, Fang H, Gair J R, Li C, Lovelace G, Mandel I and Thorne K S 2007 Phys. Rev. Lett. 99 201102-+ (Preprint arXiv:gr-qc/0612060)

[93] Rodriguez C L, Mandel I and Gair J R 2012 Phys. Rev. D. 85062002 (Preprint $1112.1404)$

[94] Smith R J E, Mandel I and Vecchio A 2013 Phys. Rev. D. 88044010 (Preprint 1302.6049)

[95] Berti E, Cardoso V and Starinets A O 2009 ArXiv e-prints (Preprint 0905.2975)

[96] Hughes S A and Menou K 2005 Astrophys.J. 623 689-699 (Preprint astro-ph/ Q410148)

[97] Ghosh A, Ghosh A, Johnson-McDaniel N K, Mishra C K, Ajith P, Del Pozzo W, Nichols D A, Chen Y, Nielsen A B, Berry C P L and London L 2016 Phys. Rev. D. 94021101 (Preprint 1602.02453)

[98] Abbott B P, Abbott R, Abbott T D, Acernese F, Ackley K, Adams C, Adams T, Addesso P, Adhikari R X, Adya V B and et al 2018 Physical Review Letters 121161101 (Preprint 1805.11581)

[99] Read J S, Markakis C, Shibata M, Uryū K, Creighton J D E and Friedman J L 2009 Phys. Rev. D. 79124033 (Preprint 0901.3258 )

[100] Hinderer T, Lackey B D, Lang R N and Read J S 2010 Phys. Rev. D. 81123016

[101] Hinderer T 2008 Astrophys. J. 6771216

[102] Vines J, Flanagan É É and Hinderer T 2011 Phys. Rev. D. 83084051 (Preprint $1101.1673)$

[103] Damour T, Nagar A and Villain L 2012 Phys. Rev. D85 123007 (Preprint 1203.4352)

[104] Read J S, Baiotti L, Creighton J D E, Friedman J L, Giacomazzo B, Kyutoku K, Markakis C, Rezzolla L, Shibata M and Taniguchi K 2013 Phys. Rev. D. 88044042 (Preprint 1306.4065)

[105] Favata M 2014 Phys. Rev. Lett. 112101101 (Preprint 1310. 8288)

[106] Wade L, Creighton J D E, Ochsner E, Lackey B D, Farr B F, Littenberg T B and Raymond V 2014 Phys. Rev. D. 89103012 (Preprint 1402.5156)

[107] Haensel P, Zdunik J L and Douchin F 2002 Astron. Astrophys. 385301 (Preprint astro-ph/0201434)

[108] Lattimer J M 2012 Ann. Rev. Nucl. Part. Sci. 62 485-515 (Preprint 1305. 3510)

[109] Perlmutter S, Aldering G, Goldhaber G, Knop R A, Nugent P, Castro P G, Deustua S, Fabbro S, Goobar A, Groom D E, Hook I M, Kim A G, Kim M Y, Lee J C, Nunes N J, Pain R, Pennypacker C R, Quimby R, Lidman C, Ellis R S, Irwin M, McMahon R G, Ruiz-Lapuente P, Walton N, Schaefer B, Boyle B J, Filippenko A V, Matheson T, Fruchter A S, Panagia N, Newberg H J M, Couch W J and Project T S C 1999 Astrophys. J. 517 565-586 (Preprint astro-ph/9812133)

[110] Komatsu E, Smith K M, Dunkley J, Bennett C L, Gold B, Hinshaw G, Jarosik N, Larson D, Nolta M R, Page L, Spergel D N, Halpern M, Hill R S, Kogut A, Limon M, Meyer S S, Odegard N, Tucker G S, Weiland J L, Wollack E and Wright E L 2011 Astrophys. J. Suppl. Ser. 19218 (Preprint 1001.4538)

[111] Planck Collaboration, Ade P A R, Aghanim N, Arnaud M, Ashdown M, Aumont J, Baccigalupi C, Banday A J, Barreiro R B, Bartlett J G and et al 2015 ArXiv e-prints (Preprint 1502.01589) 
[112] Sathyaprakash B S, Schutz B F and Van Den Broeck C 2010 Classical and Quantum Gravity 27215006 (Preprint 0906.4151 )

[113] Taylor S R and Gair J R 2012 Phys. Rev. D. 86023502 (Preprint 1204.6739)

[114] Schutz B F 1986 Nature 323 310-+

[115] Holz D E and Hughes S A 2005 Astrophys. J. 629 15-22 (Preprint arXiv: astro-ph/ Q504616)

[116] Abbott B P, Abbott R, Abbott T D, Acernese F, Ackley K, Adams C, Adams T, Addesso P, Adhikari R X, Adya V B and et al 2017 Nature 551 85-88 (Preprint 1710. 05835)

[117] Del Pozzo W 2012 Phys. Rev. D. 86043011 (Preprint 1108.1317)

[118] Messenger C and Read J 2012 Physical Review Letters 108091101 (Preprint 1107. 5725)

[119] Del Pozzo W, Li T G F and Messenger C 2017 Phys. Rev. D. 95043502 (Preprint $1506.06590)$

[120] Taylor S R, Gair J R and Mandel I 2012 Phys. Rev. D. 85023535 (Preprint 1108. 5161)

[121] Kiziltan B, Kottas A, De Yoreo M and Thorsett S E 2013 Astrophys. J. 77866 (Preprint 1011.4291)

[122] Berry C P L, Mandel I, Middleton H, Singer L P, Urban A L, Vecchio A, Vitale S, Cannon K, Farr B, Farr W M, Graff P B, Hanna C, Haster C J, Mohapatra S, Pankow C, Price L R, Sidery T and Veitch J 2015 Astrophys. J. 804114 (Preprint 1411. 6934)

[123] Misner C W, Thorne K S and Wheeler J A 1973 Gravitation (San Francisco: W.H. Freeman and Co.)

[124] Roberts L F, Ott C D, Haas R, O'Connor E P, Diener P and Schnetter E 2016 Astrophys. J. 83198 (Preprint 1604.07848)

[125] Kuroda T, Kotake K and Takiwaki T 2016 Astrophys. J. Lett. 829 L14 (Preprint 1605.09215)

[126] Takiwaki T, Kotake K and Suwa Y 2016 Mon. Not. R. Astron. Soc. 461 L112-L116 (Preprint 1602.06759)

[127] Radice D, Ott C D, Abdikamalov E, Couch S M, Haas R and Schnetter E 2016 Astrophys. J. 82076 (Preprint 1510.05022)

[128] Abdikamalov E, Ott C D, Radice D, Roberts L F, Haas R, Reisswig C, Mösta P, Klion $\mathrm{H}$ and Schnetter E 2015 Astrophys. J. 80870 (Preprint 1409. 7078)

[129] Lentz E J, Bruenn S W, Hix W R, Mezzacappa A, Messer O E B, Endeve E, Blondin J M, Harris J A, Marronetti P and Yakunin K N 2015 Astrophys. J. Lett. 807 L31 (Preprint 1505.05110)

[130] Melson T, Janka H T, Bollig R, Hanke F, Marek A and Müller B 2015 Astrophys. J. Lett. 808 L42 (Preprint 1504. 07631)

[131] Müller B and Janka H T 2015 Mon. Not. R. Astron. Soc. 448 2141-2174 (Preprint $1409.4783)$

[132] Mösta P, Ott C D, Radice D, Roberts L F, Schnetter E and Haas R 2015 Nature 528 376-379 (Preprint 1512.00838)

[133] Couch S M and O'Connor E P 2014 Astrophys. J. 785123 (Preprint 1310. 5728)

[134] Takiwaki T, Kotake K and Suwa Y 2014 Astrophys. J. 78683 (Preprint 1308. 5755)

[135] Murphy J W, Dolence J C and Burrows A 2013 Astrophys. J. 77152 (Preprint $1205.3491)$ 
[136] Hanke F, Müller B, Wongwathanarat A, Marek A and Janka H T 2013 Astrophys. J. $\mathbf{7 7 0} 66$ (Preprint 1303.6269)

[137] Couch S M 2013 Astrophys. J. 77535 (Preprint 1212.0010)

[138] Dolence J C, Burrows A, Murphy J W and Nordhaus J 2013 Astrophys. J. 765110 (Preprint 1210.5241)

[139] Ott C D, Abdikamalov E, Mösta P, Haas R, Drasco S, O'Connor E P, Reisswig C, Meakin C A and Schnetter E 2013 Astrophys. J. 768115 (Preprint 1210.6674)

[140] Müller E, Janka H T and Wongwathanarat A 2012 Astron. Astrophys. 537 A63 (Preprint 1106.6301)

[141] Takiwaki T, Kotake K and Suwa Y 2012 Astrophys. J. 74998 (Preprint 1108. 3989)

[142] Hanke F, Marek A, Müller B and Janka H T 2012 Astrophys. J. 755138 (Preprint $1108.4355)$

[143] Burrows A, Dolence J C and Murphy J W 2012 Astrophys. J. 7595 (Preprint 1204.3088)

[144] Kotake K 2011 Gravitational-wave signatures in successful vs. failed core-collapse supernovae Journal of Physics Conference Series (Journal of Physics Conference Series vol 314) p 012080

[145] Scheidegger S, Käppeli R, Whitehouse S C, Fischer T and Liebendörfer M 2010 Astron. Astrophys. 514 A51 (Preprint 1001.1570)

[146] Logue J, Ott C D, Heng I S, Kalmus P and Scargill J 2012 Phys. Rev. D. 86044023 (Preprint 1202.3256)

[147] Powell J, Gossan S E, Logue J and Heng I S 2016 Phys. Rev. D. 94123012 (Preprint 1610.05573)

[148] MacFadyen A I and Woosley S E 1999 Astrophys. J. 524 262-289 (Preprint astro-ph/9810274)

[149] Woosley S E and Bloom J S 2006 ARAA 44507

[150] Yoon S C, Langer N and Norman C 2006 Astron. Astrophys. 460 199-208 (Preprint astro-ph/0606637)

[151] Georgy C, Meynet G, Walder R, Folini D and Maeder A 2009 Astron. Astrophys. 502 611-622 (Preprint 0906.2284)

[152] Faucher-Giguère C A and Kaspi V M 2006 Astrophys. J. 643 332-355 (Preprint astro-ph/0512585)

[153] Popov S B, Pons J A, Miralles J A, Boldin P A and Posselt B 2010 Mon. Not. R. Astron. Soc. 401 2675-2686 (Preprint 0910.2190)

[154] Gullón M, Miralles J A, Viganò D and Pons J A 2014 Mon. Not. R. Astron. Soc. 443 1891-1899 (Preprint 1406.6794)

[155] Fuller J, Cantiello M, Lecoanet D and Quataert E 2015 Astrophys. J. 810101 (Preprint 1502.07779)

[156] de Mink S E, Langer N, Izzard R G, Sana H and de Koter A 2013 Astrophys. J. 764 166 (Preprint 1211.3742)

[157] Zaldarriaga M, Kushnir D and Kollmeier J A 2017 ArXiv e-prints (Preprint 1702. Q0885)

[158] Qin Y, Marchant P, Fragos T, Meynet G and Kalogera V 2019 Astrophys. J. Lett. 870 L18 (Preprint 1810.13016) 
[159] Andresen H, Müller B, Müller E and Janka H T 2017 Mon. Not. R. Astron. Soc. 468 2032-2051 (Preprint arXiv: 1607.05199)

[160] Yakunin K N, Mezzacappa A, Marronetti P, Yoshida S, Bruenn S W, Hix W R, Lentz E J, Bronson Messer O E, Harris J A, Endeve E, Blondin J M and Lingerfelt E J 2015 Phys. Rev. D. 92084040 (Preprint 1505.05824)

[161] Müller B, Janka H T and Marek A 2013 Astrophys. J. 76643 (Preprint 1210. 6984)

[162] Yakunin K N, Mezzacappa A, Marronetti P, Lentz E J, Bruenn S W, Hix W R, Messer O E B, Endeve E, Blondin J M and Harris J A 2017 ArXiv e-prints (Preprint 1701.07325)

[163] Murphy J W, Ott C D and Burrows A 2009 Astrophys. J. 707 1173-1190 (Preprint Q907.4762)

[164] Marek A, Janka H T and Müller E 2009 Astron. Astrophys. 496 475-494 (Preprint 0808.4136)

[165] Braginskii V B and Thorne K S 1987 Nature 327123

[166] Epstein R 1978 Astrophys. J. 2231037

[167] Müller E, Rampp M, Buras R, Janka H T and Shoemaker D H 2004 Astrophys. J. 603 221

[168] Ott C D 2009 Class. Quantum Grav. 26063001

[169] Kotake K, Iwakami Nakano W and Ohnishi N 2011 Astrophys. J. 736124

[170] Marek A, Janka H T and Müller E 2009 Astron. Astrophys. 496475

[171] Yakunin K N, Marronetti P, Mezzacappa A, Bruenn S W, Lee C T, Chertkow M A, Hix W R, Blondin J M, Lentz E J, Bronson Messer O E and Yoshida S 2010 Class. Quantum Grav. 27194005

[172] Müller E, Janka H T and Wongwathanarat A 2012 Astron. Astrophys. 537 A63 (Preprint 1106.6301)

[173] Müller B, Janka H T and Marek A 2013 Astrophys. J. 76643 (Preprint 1210. 6984)

[174] Obergaulinger M, Aloy M A and Müller E 2006 Astron. Astrophys. 4501107

[175] Obergaulinger M, Aloy M A, Dimmelmeier H and Müller E 2006 Astron. Astrophys. 457209

[176] Takiwaki T and Kotake K 2011 Astrophys. J. 74330 (Preprint 1004.2896)

[177] Ott C D, Burrows A, Thompson T A, Livne E and Walder R 2006 Astrophys. J. Suppl. Ser. 164130

[178] Woosley S E and Heger A 2006 Astrophys. J. 637914

[179] Heger A, Woosley S E and Spruit H C 2005 Astrophys. J. 626350

[180] Burrows A, Dessart L, Livne E, Ott C D and Murphy J 2007 Astrophys. J. 664416

[181] Takiwaki T, Kotake K and Sato K 2009 Astrophys. J. 6911360

[182] Kuroda T, Takiwaki T and Kotake K 2014 Phys. Rev. D. 89044011 (Preprint 1304. 4372)

[183] Mösta P, Richers S, Ott C D, Haas R, Piro A L, Boydstun K, Abdikamalov E, Reisswig C and Schnetter E 2014 Astrophys. J. Lett. 785 L29 (Preprint 1403. 1230)

[184] Masada Y, Takiwaki T and Kotake K 2015 Astrophys. J. Lett. 798 L22 (Preprint $1411.6705)$ 
[185] Richers S, Ott C D, Abdikamalov E, O'Connor E and Sullivan C 2017 ArXiv e-prints (Preprint 1701.02752)

[186] Dimmelmeier H, Ott C D, Marek A and Janka H T 2008 Phys. Rev. D. 78064056

[187] Abdikamalov E, Gossan S, DeMaio A M and Ott C D 2014 Phys. Rev. D. 90044001 (Preprint 1311.3678)

[188] Scheidegger S, Käppeli R, Whitehouse S C, Fischer T and Liebendörfer M 2010 Astron. Astrophys. 514 A51

[189] Ott C D, Dimmelmeier H, Marek A, Janka H T, Hawke I, Zink B and Schnetter E 2007 Phys. Rev. Lett. 98261101

[190] Fu W and Lai D 2011 Mon. Not. R. Astron. Soc. 4132207 (Preprint 1011.4887)

[191] Muhlberger C D, Nouri F H, Duez M D, Foucart F, Kidder L E, Ott C D, Scheel M A, Szilágyi B and Teukolsky S A 2014 Submitted to Phys. Rev. D.. arXiv:1405.2144 (Preprint 1405.2144)

[192] Hjorth J and Bloom J S 2011 arXiv:1 104.2274

[193] Modjaz M 2011 Astron. Nachr. 332434

[194] Woosley S E 1993 Astrophys. J. 405273

[195] MacFadyen A I, Woosley S E and Heger A 2001 Astrophys. J. 550410 (Preprint arXiv: astro-ph/9910034)

[196] Woosley S E and Bloom J S 2006 Ann. Rev. Astron. Astrophys. 44507

[197] Wheeler J C, Meier D L and Wilson J R 2002 Astrophys. J. 568807

[198] Metzger B D, Giannios D, Thompson T A, Bucciantini N and Quataert E 2011 Mon. Not. R. Astron. Soc. 4132031

[199] Piro A L and Pfahl E 2007 Astrophys. J. 6581173

[200] Korobkin O, Abdikamalov E B, Schnetter E, Stergioulas N and Zink B 2011 Phys. Rev. D. 83043007

[201] Kiuchi K, Shibata M, Montero P J and Font J A 2011 Phys. Rev. Lett. 106251102 (Preprint 1105.5035)

[202] Fryer C, Holz D and Hughes S 2002 Astrophys. J. 565430

[203] Ott C D, Reisswig C, Schnetter E, O'Connor E, Sperhake U, Löffler F, Diener P, Abdikamalov E, Hawke I and Burrows A 2011 Phys. Rev. Lett. 106161103

[204] O'Connor E and Ott C D 2011 Astrophys. J. 73070

[205] Corsi A and Mészáros P 2009 Astrophys. J. 7021171

[206] Piro A L and Ott C D 2011 Astrophys. J. 736108

[207] Ugliano M, Janka H T, Marek A and Arcones A 2012 Astrophys. J. 75769 (Preprint 1205.3657)

[208] Cerdá-Durán P, DeBrye N, Aloy M A, Font J A and Obergaulinger M 2013 Astrophys. J. Lett. 779 L18 (Preprint 1310. 8290)

[209] Ott C D, Reisswig C, Schnetter E, O'Connor E, Sperhake U, Löffler F, Diener P, Abdikamalov E, Hawke I and Burrows A 2011 Physical Review Letters 106161103 (Preprint 1012.1853)

[210] Yoon S C and Langer N 2005 Astron. Astrophys. 435967

[211] Abdikamalov E B, Ott C D, Rezzolla L, Dessart L, Dimmelmeier H, Marek A and Janka H 2010 Phys. Rev. D. 81044012 
[212] van den Bergh S and Tammann G A 1991 Ann. Rev. Astron. Astrophys. 29363

[213] Mannucci F, Della Valle M, Panagia N, Cappellaro E, Cresci G, Maiolino R, Petrosian A and Turatto M 2005 Astron. Astrophys. 433807 (Preprint arXiv:astro-ph/ Q411450)

[214] Keane E F and Kramer M 2008 Mon. Not. R. Astron. Soc. 3912009 (Preprint Q810.1512)

[215] Ando S, Beacom F and Yüksel H 2005 Phys. Rev. Lett. 95171101

[216] Kistler M D, Yüksel H, Ando S, Beacom J F and Suzuki Y 2011 Phys. Rev. D. 83 123008 (Preprint 0810. 1959)

[217] Abbott B P, Abbott R, Abbott T D, Abernathy M R, Acernese F, Ackley K, Adams C, Adams T, Addesso P, Adhikari R X and et al 2016 Phys. Rev. D. 94102001 (Preprint 1605.01785)

[218] Gossan S E, Sutton P, Stuver A et al. 2016 Phys. Rev. D. 93042002 (Preprint arXiv: 1511.02836)

[219] Hayama K, Kuroda T, Kotake K and Takiwaki T 2015 Phys. Rev. D. 92122001 (Preprint arXiv: 1501.00966)

[220] Nakamura K, Horiuchi S, Tanaka M, Hayama K, Takiwaki T and Kotake K 2016 Mon. Not. R. Astron. Soc. 461 3296-3313 (Preprint arXiv: 1602.03028)

[221] Abdikamalov E, Gossan S, DeMaio A M and Ott C D 2014 Phys. Rev. D. 90044001 (Preprint 1311.3678)

[222] Röver C, Bizouard M, Christensen N, Dimmelmeier H, Heng I S and Meyer R 2009 Phys. Rev. D. 80102004

[223] Engels W J, Frey R and Ott C D 2014 Phys. Rev. D. 90124026 (Preprint 1406.1164)

[224] Edwards M C, Meyer R and Christensen N 2014 Inverse Problems 30114008 (Preprint 1407.7549)

[225] Yokozawa T, Asano M, Kayano T, Suwa Y, Kanda N, Koshio Y and Vagins M R 2015 Astrophys. J. 81186 (Preprint 1410.2050)

[226] Halzen F and Raffelt G G 2009 Phys. Rev. D. 80087301 (Preprint 0908. 2317)

[227] Ikeda et al [Super-Kamiokande Collaboration] M 2007 Astrophys. J. 669519

[228] Abbasi et al [IceCube Collaboration] R 2011 Astron. Astrophys. 535 A109

[229] Scholberg K 2011 J. Phys. Conf. Ser. 309012028

[230] Abe K, Abe T, Aihara H, Fukuda Y, Hayato Y, Huang K, Ichikawa A K, Ikeda M, Inoue K, Ishino H, Itow Y, Kajita T, Kameda J, Kishimoto Y, Koga M, Koshio Y, Lee K P, Minamino A, Miura M, Moriyama S, Nakahata M, Nakamura K, Nakaya T, Nakayama S, Nishijima K, Nishimura Y, Obayashi Y, Okumura K, Sakuda M, Sekiya H, Shiozawa M, Suzuki A T, Suzuki Y, Takeda A, Takeuchi Y, Tanaka H K M, Tasaka S, Tomura T, Vagins M R, Wang J and Yokoyama M 2011 arXiv:1109.3262

[231] Janka H T, Langanke K, Marek A, Martínez-Pinedo G and Müller B 2007 Phys. Rep. 44238

[232] Ott C D 2010 GWs from Barmode Instabilities Tech. Rep. LIGO-T1000553-v2 LIGO Scientific Collaboration URL https: //dcc. ligo . org/LIGO-T1000553-v2

[233] Müller B, Janka H T and Heger A 2012 Astrophys. J. 76172 (Preprint 1205.7078)

[234] Ott C D, Abdikamalov E, Mösta P, Haas R, Drasco S, O'Connor E P, Reisswig C, Meakin C A and Schnetter E 2013 Astrophys. J. 768115 (Preprint 1210.6674) 
[235] Duncan R C and Thompson C 1992 Astrophys. J. Lett. 392 L9-L13

[236] Mereghetti S 2008 A E A Rev. 15 225-287

[237] Mazets E P et al. 1979 Nature 282 587-589

[238] Terasawa T, Tanaka Y T, Takei Y, Kawai N, Yoshida A, Nomoto K, Yoshikawa I, Saito Y, Kasaba Y, Takashima T, Mukai T, Noda H, Murakami T, Watanabe K, Muraki Y, Yokoyama T and Hoshino M 2005 Nature 434 1110-1111

[239] Mazets E P, Aptekar R L, Cline T L, Frederiks D D, Goldsten J O, Golenetskii S V, Hurley K, von Kienlin A, and Pal'shin V D 2008 The Astrophysical Journal 680 545549 URL http: //stacks . iop .org/0004-637X/680/545

[240] Abbott B et al. 2008 Astrophys. J. 681 1419-1430

[241] Frederiks D D, Palshin V D, Aptekar R L, Golenetskii S V, Cline T L and Mazets E P 2007 Astronomy Letters 33 19-24 (Preprint arXiv : astro-ph/0609544)

[242] Ioka K 2001 Mon. Not. R. Astron. Soc. 327 639-662 (Preprint astro-ph/0009327)

[243] Owen B J 2005 Phys. Rev. Lett. 95 211101-+ (Preprint astro-ph/0503399)

[244] Horowitz C J and Kadau K 2009 Phys. Rev. Lett. 102191102 (Preprint 0904 . 1986)

[245] Corsi A and Owen B J 2011 arXiv:1102.3421 (Preprint 1102.3421)

[246] Kashiyama K and Ioka K 2011 arXiv:1102.4830 (Preprint 1102 .4830)

[247] Levin Y and van Hoven M 2011 arXiv:astro-ph/1103.0880 (Preprint 1103.0880)

[248] Thompson C and Duncan R C 1995 Mon. Not. R. Astron. Soc. 275 255-300

[249] Benhar O, Ferrari V and Gualtieri L 2004 Phys. Rev. D. 70 124015-+ (Preprint astro-ph/0407529)

[250] McDermott P N, van Horn H M and Hansen C J 1988 Astrophys. J. 325 725-748

[251] Middleditch J, Marshall F E, Wang Q D, Gotthelf E V and Zhang W 2006 Astrophys. J. 652 1531-1546 (Preprint arXiv: astro-ph/0605007)

[252] Anderson P W and Itoh N 1975 Nature 256 25-27

[253] Andersson N, Ferrari V, Jones D I, Kokkotas K D, Krishnan B, Read J S, Rezzolla L and Zink B 2010 General relativity and gravitation 43 409-436

[254] Ushomirsky G, Cutler C and Bildsten L 2000 Mon. Not. R. Astron. Soc. 319902

[255] Kitiashvili I N and Gusev A V 2008 Astronomy Reports 52(1) 61-69 ISSN 1063-7729 10.1134/S1063772908010071 URL http://dx.doi.org/10.1134/ S1063772908010071

[256] Colaiuda A, Ferrari V, Gualtieri L and Pons J A 2008 Mon. Not. R. Astron. Soc. 385 2080

[257] Abbott B P, Abbott R, Acernese F, Adhikari R, Ajith P, Allen B, Allen G, Alshourbagy M, Amin R S, Anderson S B and et al 2010 Astrophys. J. 713671 (Preprint 0909. 3583)

[258] Abbott B, Abbott R, Adhikari R, Agresti J, Ajith P, Allen B et al. (LIGO Scientific Collaboration) 2008 Phys. Rev. D 77(2) 022001 URL http://link.aps.org/doi/ 10.1103/PhysRevD.77.022001

[259] Shibata M and Taniguchi K 2006 Phys. Rev. D. 73064027

[260] Giacomazzo B, Rezzolla L and Baiotti L 2011 Phys. Rev. D. 83044014

[261] Hotokezaka K, Kyutoku K, Okawa H, Shibata M and Kiuchi K 2011 Phys. Rev. D. 83 124008 
[262] Bauswein A, Janka H T, Hebeler K and Schwenk A 2012 Phys. Rev. D. 86063001 (Preprint 1204.1888)

[263] Cook G B, Shapiro S L and Teukolsky S A 1994 Astrophys. J. 424823

[264] Kaplan J D, Ott C D, O'Connor E P, Kiuchi K, Roberts L and Duez M 2014 Astrophys. J. 79019 (Preprint 1306.4034)

[265] Stergioulas N, Bauswein A, Zagkouris K and Janka H T 2011 Mon. Not. R. Astron. Soc. 418427 (Preprint 1105.0368)

[266] Akmal A, Pandharipande V R and Ravenhall D G 1998 Phys. Rev. C. 58 1804-1828 (Preprint nucl-th/9804027)

[267] Typel S, Röpke G, Klähn T, Blaschke D and Wolter H H 2010 Phys. Rev. C. 81015803

[268] Hempel M and Schaffner-Bielich J 2010 Nucl. Phys. A 837 210-254

[269] Shen H, Toki H, Oyamatsu K and Sumiyoshi K 1998 Nucl. Phys. A $637435-450$

[270] Lalazissis G A, König J and Ring P 1997 Phys. Rev. C. 55 540-543

[271] Hotokezaka K, Kiuchi K, Kyutoku K, Okawa H, Sekiguchi Y i, Shibata M and Taniguchi K 2013 Phys. Rev. D. 87024001 (Preprint 1212.0905)

[272] Bauswein A, Stergioulas N and Janka H T 2014 Phys. Rev. D. 90023002 (Preprint $1403.5301)$

[273] Clark J, Bauswein A, Cadonati L, Janka H T, Pankow C and Stergioulas N 2014 Phys. Rev. D. 90062004 (Preprint 1406. 5444)

[274] E T Science Team 2011 Einstein gravitational wave telescope conceptual design study Tech. rep.

[275] Allen B 1988 Phys. Rev. D 37(8) 2078-2085 URL http://link.aps.org/doi/10. 1103/PhysRevD. 37.2078

[276] Boyle L A and Steinhardt P J 2005

[277] Lehners J L and Steinhardt P J 2011 Phys. Rev. Lett. 106(8) 081301 URL http: //link.aps.org/doi/10.1103/PhysRevLett.106.081301

[278] Giblin J T and Thrane E 2014 Phys. Rev. D. 90107502 (Preprint 1410.4779)

[279] Abbott B P, Abbott R, Abbott T D, Abernathy M R, Acernese F et al. (LIGO Scientific Collaboration and Virgo Collaboration) 2017 Phys. Rev. Lett. 118(12) 121101 URL https://link.aps.org/doi/10.1103/PhysRevLett.118.121101

[280] Callister T, Sammut L, Qiu S, Mandel I and Thrane E 2016 Physical Review X 6031018 (Preprint 1604.02513)

[281] Thorne K S 1987300 Years of Gravitation ed Hawking S W and Israel W (Cambridge, UK: Cambridge University Press)

[282] Flanagan É É and Hughes S A 1998 Phys. Rev. D. 574535 